\title{
Self-Reported Visual Complaints in People with Parkinson's Disease: A Systematic Review
}

Iris van der Lijn ${ }^{a, b, *}$, Gera A. de Haan ${ }^{a, b}$, Famke Huizinga ${ }^{a, c}$, Fleur E. van der Feen ${ }^{a, b}$, A. Wijnand F. Rutgers ${ }^{\mathrm{d}}$, Catherina Stellingwerf ${ }^{\mathrm{b}}$, Teus van Laar $^{\mathrm{e}}$ and Joost Heutink ${ }^{\mathrm{a}, \mathrm{b}}$ ${ }^{a}$ Department of Clinical and Developmental Neuropsychology, University of Groningen, Groningen, the Netherlands

${ }^{\mathrm{b}}$ Royal Dutch Visio, Centre of Expertise for Blind and Partially Sighted People, Huizen, the Netherlands ${ }^{\mathrm{c}}$ Department of General Practice and Elderly Care Medicine, University Medical Center Groningen, Groningen, the Netherlands

${ }^{\mathrm{d}}$ Department of Neurology, Martini Ziekenhuis Groningen, Groningen, the Netherlands

${ }^{\mathrm{e}}$ Department of Neurology, University of Groningen, University Medical Centre Groningen, Groningen, the Netherlands

Accepted 17 December 2021

Pre-press 4 January 2022

\begin{abstract}
.
Background: Scientific research increasingly focuses on visual symptoms of people with Parkinson's disease (PD). However, this mostly involves functional measures, whereas self-reported data are equally important for guiding clinical care.

Objective: This review provides an overview of the nature and prevalence of self-reported visual complaints by people with PD, compared to healthy controls.

Methods: A systematic literature search was performed. Studies from three databases (PubMed, PsycInfo, and Web of Science) were screened for eligibility. Only studies that reported results of visual self-reports in people with idiopathic PD were included.

Results: One hundred and thirty-nine eligible articles were analyzed. Visual complaints ranged from function-related complaints (e.g., blurred vision, double vision, increased sensitivity to light or changes in contrast sensitivity) to activity-related complaints (e.g., difficulty reading, reaching, or driving). Visual complaints were more prevalent in people with PD compared to healthy controls. The presence of visual complaints leads to a reduced quality of life (QoL). Increased prevalence and severity of visual complaints in people with PD are related to longer disease duration, higher disease severity, and off-state. Conclusion: A large proportion of people with PD have visual complaints, which negatively affect QoL. Complaints are diverse in nature, and specific and active questioning by clinicians is advised to foster timely recognition, acknowledgement, and management of these complaints.
\end{abstract}

Keywords: Activities of daily living, Parkinson's disease, quality of life, self report, systematic review, vision disorders

\footnotetext{
${ }^{*}$ Correspondence to: Iris van der Lijn, Clinical and Developmental Neuropsychology, University of Groningen, Grote Kruisstraat 1/2, 9712 TS Groningen, the Netherlands. Tel.: +3150 3636311; E-mail: i.van.der.lijn@rug.nl.
} 


\section{INTRODUCTION}

Parkinson's disease (PD) is a neurodegenerative disorder characterized by a number of well-known motor and non-motor symptoms, including visual problems [1-5]. During recent years, visual symptoms in PD have received increasing attention in the literature. In clinical practice however, these symptoms are still underrecognized. Visual symptoms are less conspicuous than motor symptoms, and may therefore be overlooked [6, 7]. However, visual symptoms have a substantial impact on the daily lives of people with PD and may already occur in the early and prodromal stages of the disease [1, 4, 8]. Consequently, several studies state the importance of tools to improve the recognition, diagnostic accuracy, and management of non-motor symptoms, including visual symptoms, in clinical practice [2, 9-11].

To date, most tools used to assess visual symptoms are quantitative tests of visual functions. These measurements, in most cases part of an ophthalmological assessment, objectify functions and impairments, such as visual acuity and visual field loss. In contrast, few studies include self-report data, asking people to reflect on their own visual functioning. It is acknowledged that both functional and self-reported data are required to obtain a clear picture of the impact of PD on individuals [9], which is necessary to guide care and rehabilitation $[12,13]$.

Despite the importance of self-reported data, no reviews have been published on this topic so far. Therefore, this paper presents a systematic review on self-reported visual complaints in PD, compared to healthy controls.

\section{METHODS}

\section{Literature search}

A literature search was performed according to the guidelines of Preferred Reporting Items for Systematic Reviews and Meta-Analyses (PRISMA) [14]. The search encompassed the scientific databases PsycInfo, PubMed, and Web of Science (Core Collection) and was performed on literature up to the date of February 5, 2021. The search terms are presented in Table 1. Two complementary searches were performed. The first one focused on subjective dysfunction in general, since the current review was part of a project that also examined self-reported cognitive complaints of people with PD. The second search addressed self-reported visual complaints specifically.

\section{Eligibility criteria}

Only papers fulfilling the following criteria were included: written in English, published in peerreviewed academic journals, including human participants with idiopathic PD, including a self-report measure for visual functioning, and data (i.e., prevalence) of this measure for the group of people with PD or a comparison (i.e., prevalence or score) with healthy controls. Visual self-report measures were defined as items, subscales or total scores representing an individual's reflection on their own visual functioning, performance of visual activities or vision-related quality of life $(\mathrm{QoL})$. We made the assumption that a self-report measure was filled out by the patient, unless otherwise stated. Longitudinal or intervention studies were included if relevant baseline measures were presented. Review articles and case reports were excluded, as well as meeting abstracts and letters to editors. Since visual hallucinations in PD are widely recognized and extensively studied [15], these were not incorporated in this review.

\section{Literature extraction}

Figure 1 shows the PRISMA outline of papers identified through the literature search. Articles were first screened by title and abstract, and when they seemed relevant, reviewed by the full text. In addition to the database search, reference lists of included papers were screened according to the predefined inclusion criteria. Two authors (IvdL and FH) and a research assistant (EV) systematically judged the eligibility of articles and discussed this in case of doubt. In case uncertainty remained, a third author $(\mathrm{GdH})$ was involved until consensus was reached.

\section{Analysis}

Relevant results were extracted from included papers. Results were categorized per complaint. Complaints were divided amongst three categories: function-related complaints, activity-related complaints, and vision-related QoL. Results were ordered based on the number of studies investigating each complaint. Based on this systematic categorization, a descriptive table was included (Table 2). If data on subgroups of PD were reported (e.g., tremor dominant versus akinetic-rigidity subtypes, or young versus late onset PD), but no total prevalence, it was calculated by the authors. The weighted prevalence of a complaint was calculated for studies using the 
Table 1

Search terms for literature search

\begin{tabular}{|c|c|}
\hline Search 1 & Terms used in all databases \\
\hline $\begin{array}{l}\text { First } \\
\text { criterion }\end{array}$ & parkinson*a \\
\hline $\begin{array}{l}\text { Second } \\
\text { criterion }\end{array}$ & 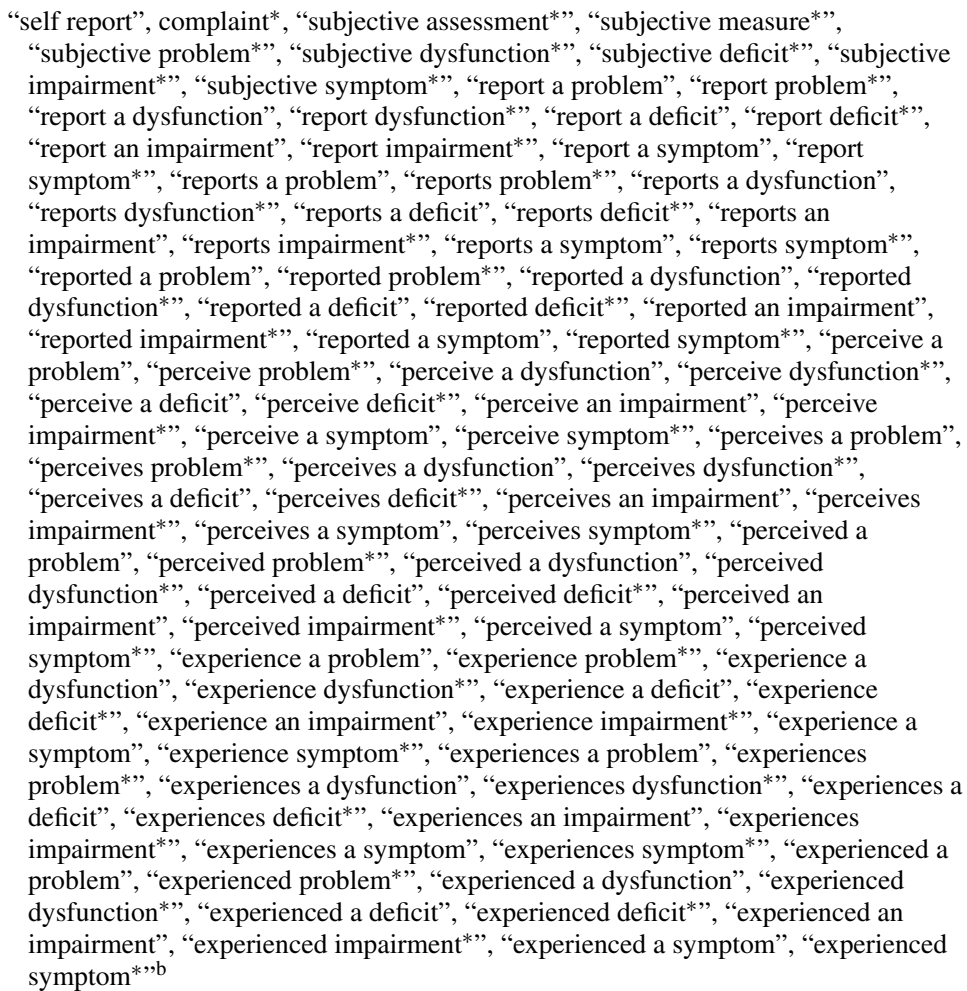 \\
\hline
\end{tabular}

\begin{tabular}{ll}
\hline Search 2 & Terms used in all databases \\
\hline $\begin{array}{c}\text { First } \\
\text { criterion }\end{array}$ & parkinson*a
\end{tabular}

Second questionnaire*, scale $^{*}$, survey*, inventor*, interview ${ }^{* b}$

criterion

Third seeing OR eyesight OR sight OR ophthalmologic* OR "visual perception" OR "visual criterion acuity" OR "visual field"” OR diplopia OR "double vision" OR "depth perception" OR "color vision" OR "colour vision" OR "color perception" OR "colour perception" OR "contrast sensitivity" OR "visual contrast” OR "brightness perception" OR "light sensitivity" OR "sensitive to light" OR "sensitivity to light" OR "oversensitive to light" OR "oversensitivity to light" OR photophobia OR photosensitivity OR glare OR "dark adaptation" OR "visual overload" OR "problems reading" OR "reading problem*" OR "difficulty reading” OR "reading difficult*" OR "painful eyes" OR "dry eyes" OR "eyelid opening" OR blink* OR "facial recognition" OR "face recognition" OR "face perception" OR "object recognition" OR "object perception" OR "figure perception" OR "form perception" OR "shape perception" OR "motion perception" OR "space perception" OR "spatial perception" OR "spatial orientation" OR "spatial disorientation" OR afterimage* OR “visual discrimination" OR "figure ground discrimination" OR "nonmotor symptom*" OR "non-motor symptom*" OR "autonomic dysfunction*" OR "autonomic symptom*" OR "autonomous dysfunction" OR "autonomous
$\mathrm{MeSH}$ and thesaurus terms used in PubMed or PsycInfo

PubMed: "Parkinson Disease";

PsycInfo: "Parkinson's Disease"

Pubmed: "Self Report"; PsycInfo: "Self Report", "Self-Report"
$\mathrm{MeSH}$ and thesaurus terms used in PubMed or PsycInfo

PubMed: "Parkinson Disease"; PsycInfo: "Parkinson's Disease"

PubMed: "Surveys and Questionnaires", "Interview, Psychological"; PsycInfo: Questionnaires, Interviews, Surveys, Inventories PubMed: "Visual Perception", "Vision disorders", "Vision screening", "Depth Perception", "Color vision"; PsycInfo: "Visual perception", "Vision disorders", "Diplopia", "Depth perception", "Color perception", "Brightness perception", "Dark adaptation", "Face perception", "Visual acuity", "Visual contrast", "Visual discrimination", "Visual field", "Figure ground discrimination" symptom*",b

\footnotetext{
${ }^{a}$ Term should be present in title or abstract, ${ }^{b}$ One of the terms should be present in the content of the paper.
} 


\section{PRISMA PRISMA 2009 Flow Diagram}
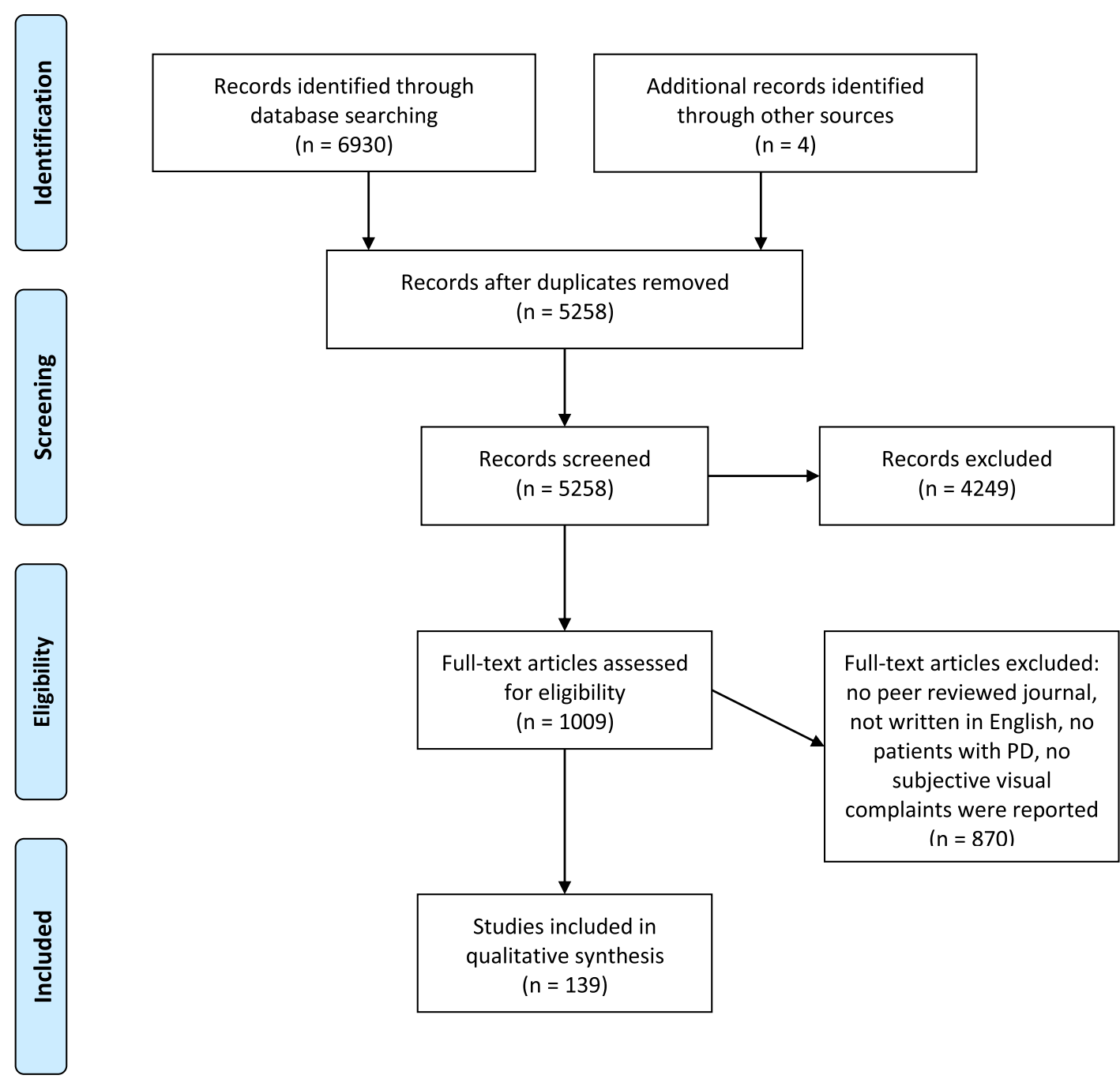

Full-text articles excluded: no peer reviewed journal, not written in English, no patients with PD, no subjective visual complaints were reported ( $n=870)$

Fig. 1. PRISMA flow diagram of systematic literature search [14].

same method to assess the complaint. Relationships of self-reported visual complaints with demographics and disease-related variables were gathered in an additional table (Table 3).

\section{RESULTS}

The search procedure resulted in 139 relevant articles. Table 2 presents the prevalence of visual complaints reported by people with PD and the comparison with healthy controls. One hundred and thirty-one studies investigated the prevalence of visual complaints in people with PD. Some studies reported on more than one complaint, resulting in 185 prevalence numbers. Forty-four studies compared the presence of visual complaints between people with PD and healthy controls. Some studies reported group differences for multiple complaints, resulting in 122 numbers on group comparisons. Of these, 102 $(83.6 \%)$ indicated that people with PD reported more complaints than healthy controls, whilst 20 (16.4\%) found either no difference between these groups or reported healthy controls to experience more complaints than people with PD. 
Table 2

Overview of the prevalence of visual complaints in people with PD and the comparison to healthy controls

\begin{tabular}{|c|c|c|c|c|c|c|c|c|c|}
\hline & $\begin{array}{l}\text { Items } \\
\text { covering } \\
\text { complaint } \\
(N)\end{array}$ & Criterion presence complaint & $\begin{array}{l}\text { Studies } \\
(N)\end{array}$ & $\begin{array}{c}\text { PD } \\
\text { patients } \\
(N)\end{array}$ & $\begin{array}{c}\text { Prevalence } \\
\text { PD patients } \\
(\%)\end{array}$ & $\begin{array}{l}\text { Controls } \\
(N)\end{array}$ & $\begin{array}{l}\text { Prevalence } \\
\text { controls } \\
(\%)\end{array}$ & $\begin{array}{c}\text { Studies } \\
\text { PD }>\text { Controls }^{\mathrm{a}} \\
(N)\end{array}$ & $\begin{array}{c}\text { Studies } \\
\mathrm{PD}=\text { Controls } \\
\text { or } \\
\mathrm{PD}<\text { Controls } \\
(N) \\
\end{array}$ \\
\hline \multicolumn{10}{|l|}{ Function-related visual complaints } \\
\hline Visual complaints in general & & & 17 & 2337 & $26.8-98$ & 29344 & $16.1-31.6$ & 6 & 0 \\
\hline CIRS-G & 1 & $\begin{array}{l}\geq 1 \text { on a } 0 \text { ('no problem') }-4 \\
\text { ('extremely severe') scale }\end{array}$ & $1[19]$ & 76 & 98 & N/A & N/A & N/A & N/A \\
\hline VFQ-25 & 25 & At least one complaint & $3[18,25,26]$ & 145 & $83.1[25,26]$ & 466 & $31.6[25]$ & 3 & 0 \\
\hline $\begin{array}{l}\text { Direct asking of the patient } \\
\text { according to ICF checklist }\end{array}$ & 2 & $\begin{array}{l}\geq 1 \text { ('mild difficulty') on a } 0- \\
4 \text { scale }\end{array}$ & $1[27]$ & 96 & $75.5^{\mathrm{b}}$ & N/A & N/A & N/A & N/A \\
\hline $\begin{array}{l}\text { Interview on autonomic } \\
\text { dysfunction }\end{array}$ & 4 & N/A & $1[20]$ & 26 & 67 & 27 & 22 & 1 & 0 \\
\hline $\begin{array}{l}\text { Questionnaire on visual } \\
\text { complaints (20-item) }\end{array}$ & N/A & N/A & $1[16]$ & 30 & 63.3 & 31 & 29.0 & 1 & 0 \\
\hline PRO-PD & 1 & $\begin{array}{l}\geq 5 \text { on a } 0 \text { ('lack of } \\
\text { symptom') - } 100 \\
\text { ('maximum severity') scale }\end{array}$ & $1[32]$ & 1031 & 58.0 & N/A & N/A & N/A & N/A \\
\hline Self-report questionnaire (31-item) & N/A & At least one complaint & $1[17]$ & 81 & $78^{\mathrm{c}}$ & N/A & N/A & N/A & N/A \\
\hline $\begin{array}{l}\text { Survey of Health, Ageing, and } \\
\text { Retirement in Europe }\end{array}$ & 1 & $\begin{array}{l}\geq 4 \text { on a } 1 \text { ('excellent') }-5 \\
\text { ('poor') scale }\end{array}$ & $1[21]$ & 191 & 46.1 & 28820 & 16.1 & 1 & 0 \\
\hline $\begin{array}{l}\text { Interview using a questionnaire on } \\
\text { non-motor symptoms [30] }\end{array}$ & 1 & 'yes' & $2[22,30]$ & 76 & 46.1 & N/A & N/A & N/A & N/A \\
\hline Symptom questionnaire & 1 & 'yes' & $1[28]$ & 39 & 43.6 & N/A & N/A & N/A & N/A \\
\hline $\begin{array}{l}\text { Question: are you bothered due to } \\
\text { vision-related problems? }\end{array}$ & 1 & N/A & $1[23]$ & 295 & 41 & N/A & N/A & N/A & N/A \\
\hline Structured interview & N/A & N/A & $1[29]$ & 108 & 40.7 & N/A & N/A & N/A & N/A \\
\hline Symptom index (20-item) & 1 & 'yes' & $1[31]$ & 61 & 36.1 & N/A & N/A & N/A & N/A \\
\hline Questions on visual complaints & N/A & N/A & $1[24]$ & 82 & 26.8 & N/A & N/A & N/A & N/A \\
\hline Double vision & & & 96 & 20994 & $7.7-53.1$ & 1556 & $2.4-19.0$ & 15 & 3 \\
\hline Questionnaire on changes in vision & 1 & 'sometimes' or 'often' & $2[118,119]$ & 237 & 53.1 & 100 & 19.0 & 2 & 0 \\
\hline Self-report questionnaire (31-item) & 1 & 'sometimes' or 'often' & $1[17]$ & 81 & 38.0 & N/A & N/A & N/A & N/A \\
\hline VIPD-Q & 1 & $\begin{array}{l}\geq 2 \text { ('weekly') on a } 0 \\
\text { ('never') - } 3 \text { ('daily') scale }\end{array}$ & $1[117]$ & 848 & 22 & 250 & 3 & 1 & 0 \\
\hline $\begin{array}{l}\text { Questionnaire derived from } \\
\text { VFQ-25 }\end{array}$ & N/A & N/A & $2[25,116]$ & 178 & 19.8 & 122 & 2.4 & 2 & 0 \\
\hline $\begin{array}{l}\text { Non-motor fluctuations } \\
\text { questionnaire }\end{array}$ & 1 & N/A & $1[159]$ & 11 & 18.2 & N/A & N/A & N/A & N/A \\
\hline NMSQ & 1 & $\geq 1$ ('yes') & 61 [33-93] & 11511 & $13.9^{\mathrm{d}}$ & 690 & $\begin{array}{l}3.5[40,47 \\
48,56,57 \\
59,91,92]\end{array}$ & $\begin{array}{c}6[47,48,56 \\
57,59,91]\end{array}$ & $2[40,92]$ \\
\hline
\end{tabular}


Table 2

(Continued)

\begin{tabular}{|c|c|c|c|c|c|c|c|c|c|}
\hline & $\begin{array}{l}\text { Items } \\
\text { covering } \\
\text { complaint } \\
(N)\end{array}$ & Criterion presence complaint & $\begin{array}{l}\text { Studies } \\
(N)\end{array}$ & $\begin{array}{c}\text { PD } \\
\text { patients } \\
(N)\end{array}$ & $\begin{array}{c}\text { Prevalence } \\
\text { PD patients } \\
(\%)\end{array}$ & $\begin{array}{l}\text { Controls } \\
(N)\end{array}$ & $\begin{array}{c}\text { Prevalence } \\
\text { controls } \\
(\%)\end{array}$ & $\begin{array}{c}\text { Studies } \\
\text { PD }>\text { Controls }{ }^{\mathrm{a}} \\
(N)\end{array}$ & $\begin{array}{c}\text { Studies } \\
\mathrm{PD}=\text { Controls } \\
\text { or } \\
\mathrm{PD}<\text { Controls } \\
(N) \\
\end{array}$ \\
\hline NMSS & 1 & $\begin{array}{l}\geq 1 \text { ('mild') on a } 0 \text { ('none') - } \\
3 \text { ('severe') scale }\end{array}$ & $24[9,41,94-115]$ & 7545 & $\begin{array}{c}14.2[9,41 \\
94,96-107, \\
109-115]\end{array}$ & 363 & $2.4[94,96]$ & $3[95,96,108]$ & $1[94]$ \\
\hline Online symptom survey & 1 & 'yes' & $1[160]$ & 432 & 12.7 & N/A & N/A & N/A & N/A \\
\hline $\begin{array}{l}\text { Questionnaire on visual } \\
\text { complaints (20-item) }\end{array}$ & N/A & N/A & $1[16]$ & 30 & 10.0 & 31 & 3.2 & 1 & 0 \\
\hline Questions on visual complaints & N/A & N/A & $1[24]$ & 82 & 9.8 & N/A & N/A & N/A & N/A \\
\hline Questions on asthenopia & N/A & N/A & $1[152]$ & 39 & 7.7 & N/A & N/A & N/A & N/A \\
\hline Increased sensitivity to light & & & 27 & 6318 & $15.0-48$ & 1480 & $0-28.3$ & 13 & 2 \\
\hline $\begin{array}{l}\text { Interview on autonomic } \\
\text { dysfunction }\end{array}$ & 1 & N/A & $1[20]$ & 26 & 48 & N/A & N/A & N/A & N/A \\
\hline VIPD-Q & 1 & $\begin{array}{l}\geq 2 \text { ('weekly') on a } 0 \\
\text { ('never') - } 3 \text { ('daily') scale }\end{array}$ & $1[117]$ & 848 & 43 & 250 & 23 & 1 & 0 \\
\hline $\begin{array}{l}\text { ADS (autonomic dysfunction } \\
\text { score) }\end{array}$ & 1 & N/A & $1[140]$ & 21 & 38.1 & 27 & 0 & 1 & 0 \\
\hline SCOPA-AUT & 1 & $\begin{array}{l}\geq 1 \text { ('sometimes') on a } 0 \\
\quad \text { ('never') }-3 \text { ('often') scale }\end{array}$ & 19 [120-138] & 5195 & $\begin{array}{c}31.8[120,122, \\
124-130 \\
132-138]\end{array}$ & 1044 & $\begin{array}{l}28.3[120, \\
128-130, \\
132,138]\end{array}$ & $\begin{array}{c}8[120,121, \\
123,128-130 \\
132,138]\end{array}$ & $1[131]$ \\
\hline COMPASS-31 & 5 & N/A & $1[161]$ & 47 & 27.7 & N/A & N/A & N/A & N/A \\
\hline $\begin{array}{l}\text { Questionnaire derived from } \\
\text { VFQ-25 }\end{array}$ & N/A & N/A & $1[25]$ & 88 & 23.9 & 90 & 11.8 & 1 & 0 \\
\hline Questionnaire on changes in vision & 1 & 'sometimes' or 'often' & $1[119]$ & 53 & 15 & 31 & 3 & 1 & 0 \\
\hline VAQ & 3 & N/A & $1[142]$ & 25 & N/A & 23 & N/A & 0 & 1 \\
\hline COMPASS & 7 & N/A & $1[139]$ & 15 & N/A & 15 & N/A & 1 & 0 \\
\hline Altered color vision & & & 9 & 1394 & $4.1-12.7$ & 894 & $3-10$ & 4 & 4 \\
\hline Questionnaire on changes in vision & 1 & 'sometimes' or 'often' & $2[118,119]$ & 237 & 12.7 & 100 & 4.4 & $1[118]$ & $1[119]$ \\
\hline VIPD-Q & 1 & $\begin{array}{l}\geq 2 \text { ('weekly') on a } 0 \\
\text { ('never') - } 3 \text { ('daily') scale }\end{array}$ & $1[117]$ & 848 & 11 & 250 & 3 & 1 & 0 \\
\hline Questions on visual complaints & N/A & N/A & $1[24]$ & 82 & 6.1 & N/A & N/A & N/A & N/A \\
\hline Interview on prodromal symptoms & 1 & 'yes' & $2[141,143]$ & 145 & 4.1 & 145 & 10 & 0 & 2 \\
\hline VFQ-25 & 1 & N/A & $2[18,26]$ & 57 & N/A & 376 & N/A & 2 & 0 \\
\hline VAQ & 3 & N/A & $1[142]$ & 25 & N/A & 23 & N/A & 0 & 1 \\
\hline
\end{tabular}


Painful/dry eyes

Dry eye symptom assessment

VIPD-Q

Question on dry eye

VFQ-25

Online symptom survey (dry eyes)

Questions on visual complaints

OSDI

Blurred vision

VIPD-Q

Symptom questionnaire

ADS (autonomic dysfunction

score)

Interview on visual symptoms

Online symptom survey

Questionnaire derived from

VFQ-25

Changes in contrast sensitivity

Questionnaire on changes in vision

VIPD-Q

Interview on visual symptoms

Changes in size and shape perception

Questionnaire on changes in vision

VIPD-Q

Questions on visual complaints

Changes in depth perception

Questionnaire on changes in vision

VIPD-Q

VAQ

Reduced peripheral vision

VFQ-25

VAQ

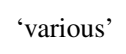

'sometimes' or 'often'

$\geq 2$ ('weekly') on a 0

('never') - 3 ('daily') scale 'yes'

'sometimes' or 'often'

$\geq 2$ ('weekly') on a 0

('never') - 3 ('daily') scale

N/A N/A

'sometimes' or 'often

$\geq 2$ ('weekly') on a 0

('never') - 3 ('daily') scale

N/A

N/A

N/A

\begin{tabular}{|c|c|c|}
\hline 9 & 1706 & $6.1-87.5$ \\
\hline 1 [144] & 56 & 87.5 \\
\hline 1 [117] & 848 & 28 \\
\hline 1 [162] & 43 & 44.2 \\
\hline $3[18,25,26]$ & 145 & $27.3[25]$ \\
\hline $1[160]$ & 432 & 25.7 \\
\hline $1[24]$ & 82 & 6.1 \\
\hline 1 [145] & 100 & N/A \\
\hline 6 & 1695 & $19.7-46$ \\
\hline 1 [117] & 848 & 46 \\
\hline 1 [146] & 181 & 36.2 \\
\hline $1[140]$ & 21 & 28.6 \\
\hline 1 [111] & 125 & 20 \\
\hline $1[160]$ & 432 & 19.7 \\
\hline 1 [25] & 88 & N/A \\
\hline 3 & 1157 & $8.8-27$ \\
\hline 1 [118] & 184 & 27 \\
\hline 1 [117] & 848 & 26 \\
\hline 1 [111] & 125 & 8.8 \\
\hline 3 & 982 & $3.7-19$ \\
\hline 1 [119] & 53 & $19^{\mathrm{g}}$ \\
\hline 1 [117] & 848 & 17 \\
\hline $1[24]$ & 82 & 3.7 \\
\hline 3 & 926 & $17-26$ \\
\hline 1 [119] & 53 & 26 \\
\hline 1 [117] & 848 & 17 \\
\hline 1 [142] & 25 & N/A \\
\hline 3 & 82 & N/A \\
\hline $2[18,26]$ & 57 & N/A \\
\hline 1 [142] & 25 & N/A \\
\hline
\end{tabular}

$8-22.4$
20.6

N/A

22.4 [25]

N/A
$2[18,25]$

N/A

N/A

N/A

$1[26]$

N/A

$7.4-19.0$

N/A

12

19.0

7.4

N/A

N/A

$10-13$

13
10

N/A

$5-13$
13

5

N/A

2-6

2

N/A

N/A

N/A

N/A 
Table 2

(Continued)

\begin{tabular}{|c|c|c|c|c|c|c|c|c|c|}
\hline & $\begin{array}{c}\text { Items } \\
\text { covering } \\
\text { complaint } \\
(N)\end{array}$ & Criterion presence complaint & $\begin{array}{l}\text { Studies } \\
(N)\end{array}$ & $\begin{array}{c}\text { PD } \\
\text { patients } \\
(N)\end{array}$ & $\begin{array}{c}\text { Prevalence } \\
\text { PD patients } \\
(\%)\end{array}$ & $\begin{array}{l}\text { Controls } \\
(N)\end{array}$ & $\begin{array}{c}\text { Prevalence } \\
\text { controls } \\
(\%)\end{array}$ & $\begin{array}{c}\text { Studies } \\
\text { PD }>\text { Controls }^{\mathrm{a}} \\
(N)\end{array}$ & $\begin{array}{c}\text { Studies } \\
\mathrm{PD}=\text { Controls } \\
\text { or } \\
\mathrm{PD}<\text { Controls } \\
(N) \\
\end{array}$ \\
\hline Difficulty estimating spatial relations & & & 3 & 390 & $6.4-50$ & 69 & 28 & 1 & 0 \\
\hline Questionnaire on changes in vision & 1 & 'sometimes' or 'often' & $1[118]$ & 184 & 50 & 69 & 28 & 1 & 0 \\
\hline Self-report questionnaire (31-item) & 1 & 'sometimes' or 'often' & $1[17]$ & 81 & 45.0 & N/A & N/A & N/A & N/A \\
\hline Interview on visual symptoms & 1 & ‘yes’ & $1[111]$ & 125 & 6.4 & N/A & N/A & N/A & N/A \\
\hline Visual field impairment & & & 2 & 930 & $6.1-9$ & 250 & 2 & 1 & 0 \\
\hline VIPD-Q & 1 & $\begin{array}{l}\geq 2 \text { ('weekly') on a } 0 \\
\quad \text { ('never') - 3 ('daily') scale }\end{array}$ & $1[117]$ & 848 & 9 & 250 & 2 & 1 & 0 \\
\hline Questions on visual complaints & N/A & N/A & $1[24]$ & 82 & 6.1 & N/A & N/A & N/A & N/A \\
\hline Watery eyes & & & 2 & 930 & $26.8-31$ & 250 & 15 & 1 & 0 \\
\hline VIPD-Q & 1 & $\begin{array}{l}\geq 2 \text { ('weekly') on a } 0 \\
\quad \text { ('never') - 3 ('daily') scale }\end{array}$ & $1[117]$ & 848 & 31 & 250 & 15 & 1 & 0 \\
\hline Questions on visual complaints & N/A & N/A & $1[24]$ & 82 & 26.8 & N/A & N/A & N/A & N/A \\
\hline Changes in motion perception & & & 2 & 237 & 23.7 & 100 & 3 & 2 & 0 \\
\hline Questionnaire on changes in vision & 1 & ‘sometimes' or ‘often’ & $2[118,119]$ & 237 & 23.7 & 100 & 3 & 2 & 0 \\
\hline Reduced night vision & & & 2 & 869 & $16-47.6$ & 277 & $3-18.5$ & 2 & 0 \\
\hline $\begin{array}{l}\text { ADS (autonomic dysfunction } \\
\text { score) }\end{array}$ & 1 & N/A & $1[140]$ & 21 & 47.6 & 27 & 18.5 & 1 & 0 \\
\hline VIPD-Q & 1 & $\begin{array}{l}\geq 2 \text { ('weekly') on a } 0 \\
\text { ('never') - 3 ('daily') scale }\end{array}$ & $1[117]$ & 848 & 16 & 250 & 3 & 1 & 0 \\
\hline Reduced light/dark adaptation & & & 2 & 873 & 27 & 273 & 11 & 2 & 1 \\
\hline VIPD-Q & 1 & $\begin{array}{l}\geq 2 \text { ('weekly') on a } 0 \\
\quad \text { ('never') - 3 ('daily') scale }\end{array}$ & 1 [117] & 848 & 27 & 250 & 11 & 1 & 0 \\
\hline VAQ & 4 & N/A & $1[142]$ & 25 & N/A & 23 & N/A & $1^{\mathrm{e}}$ & $1^{\mathrm{f}}$ \\
\hline Floaters & & & 2 & 178 & N/A & 122 & N/A & 0 & 2 \\
\hline $\begin{array}{l}\text { Questionnaire derived from } \\
\text { VFQ-25 }\end{array}$ & N/A & N/A & $2[25,116]$ & 178 & N/A & 122 & N/A & 0 & 2 \\
\hline Misjudge objects & & & 2 & 178 & N/A & 122 & N/A & 2 & 0 \\
\hline $\begin{array}{l}\text { Questionnaire derived from } \\
\text { VFQ-25 }\end{array}$ & N/A & N/A & $2[25,116]$ & 178 & N/A & 122 & N/A & 2 & 0 \\
\hline Asthenopia (ocular fatigue) & & & 1 & 39 & 43.6 & 39 & 5.1 & 1 & 0 \\
\hline Questions on asthenopia & N/A & $\begin{array}{l}\text { Presence of visual discomfort, } \\
\text { diplopia, blurred vision or } \\
\text { headache when reading }\end{array}$ & $1[152]$ & 39 & 43.6 & 39 & 5.1 & 1 & 0 \\
\hline
\end{tabular}


Hard to follow quick movements with the eyes

$$
\text { VIPD-Q }
$$

$\geq 2$ ('weekly') on a 0

$1 \quad 848$

848

25

250

('never') - 3 ('daily') scale

1 [117] 848

Need to turn head to side to see

153

peripheral objects

Questionnaire on changes in vision

'sometimes' or 'often'

1 [119]

43

31

Mucus/slime or particles in eyes or

eyelids

VIPD-Q

Reduced acuity/spatial vision

$$
\text { VAQ }
$$

Reduced visual processing speed

VAQ

Reduced visual search

$$
\text { VAQ }
$$

Activity-related visual complaints

Reading difficulties

Self-report questionnaire (31-item)

Questionnaire on visual

complaints (20-item)

Questions on asthenopia

VIPD-Q

Questionnaire derived from

$$
\text { VFQ-25 }
$$

Near activities

Survey of Health, Ageing, and

Retirement in Europe

CISS

VFQ-25

Difficulty/freezing in narrow spaces

Questionnaire on changes in vision

Questionnaire derived from

VFQ-25

Distance activities

Survey of Health, Ageing, and

Retirement in Europe

$\geq 2$ ('weekly') on a 0

('never') - 3 ('daily') scale

1 [117] 848

125

1 [142] 25

$1-25$

1 [142] 25

$1 \quad 25$

$1[142] \quad 25$

$6 \quad 1176$

$\begin{array}{cc}6 & 1176 \\ 1[17] & 81\end{array}$

30

N/A N/A

$1[16$

1 [152] 39

1 [117] 848

$\geq 2$ ('weekly') on a 0

$2[25,116] \quad 178$

N/A

$5 \quad 1875$

$\begin{array}{cc}5 & 1875 \\ 1[21] & 1438\end{array}$

$\geq 4$ on a 1 ('excellent') -5

('poor') scale

$14 \geq 21$

$3 \quad$ N/A

1 'sometimes' or 'often'

N/A N/A

$\begin{array}{ccccccc}2[147,148] & 380 & 34.5 & 380 & 9.7 & 2 & 0 \\ 2[18,26] & 57 & \text { N/A } & 376 & \text { N/A } & 2 & 0\end{array}$

$4 \quad 415$

$2[118,119] \quad 237$

$2[25,116] \quad 178$

67.3

$67.3^{\mathrm{i}}$

N/A

222

$3 \quad 1495$

1 [21] 1438

38.6

38.6

122

1.6

$1.6^{\mathrm{i}}$

N/A

$2[18,26] \quad 57$

N/A

376

N/A

2

Continued) 
Table 2

Continued

\begin{tabular}{|c|c|c|c|c|c|c|c|c|c|}
\hline & $\begin{array}{c}\text { Items } \\
\text { covering } \\
\text { complaint } \\
(N)\end{array}$ & Criterion presence complaint & $\begin{array}{l}\text { Studies } \\
(N)\end{array}$ & $\begin{array}{c}\mathrm{PD} \\
\text { patients } \\
(N)\end{array}$ & $\begin{array}{c}\text { Prevalence } \\
\text { PD patients } \\
(\%)\end{array}$ & $\begin{array}{l}\text { Controls } \\
(N)\end{array}$ & $\begin{array}{l}\text { Prevalence } \\
\text { controls } \\
(\%)\end{array}$ & $\begin{array}{c}\text { Studies } \\
\text { PD }>\text { Controls }{ }^{\mathrm{a}} \\
(N)\end{array}$ & $\begin{array}{c}\text { Studies } \\
\mathrm{PD}=\text { Controls } \\
\text { or } \\
\mathrm{PD}<\text { Controls }^{\mathrm{a}} \\
(N)\end{array}$ \\
\hline Driving & & & 3 & 94 & 21.6 & 355 & 2.7 & 3 & 0 \\
\hline VFQ-25 & 2 & N/A & $2[18,26]$ & 57 & N/A & 318 & N/A & 2 & 0 \\
\hline $\begin{array}{l}\text { Self-report driving questionnaire } \\
\text { derived from Charlton et al. } \\
\text { (2006) [163] }\end{array}$ & N/A & $\begin{array}{l}\text { ‘very difficult' in low } \\
\text { visibility conditions }\end{array}$ & 1 [149] & 37 & 21.6 & 37 & 2.7 & 1 & 0 \\
\hline Difficulty reaching for objects & & & 1 & 184 & 46 & 69 & 3 & 1 & 0 \\
\hline Questionnaire on changes in vision & 1 & 'sometimes' or 'often' & $1[118]$ & 184 & 46 & 69 & 3 & 1 & 0 \\
\hline Problems with visual ADLs & & & 1 & 25 & N/A & 23 & N/A & 1 & 1 \\
\hline VAQ & 33 & N/A & $1[142]$ & 25 & N/A & 23 & N/A & $1^{\mathrm{e}}$ & $1^{\mathrm{f}}$ \\
\hline \multicolumn{10}{|l|}{ Vision-related QoL } \\
\hline Vision-specific social functioning & & & 2 & 57 & N/A & 376 & N/A & 2 & 0 \\
\hline VFQ-25 & 2 & N/A & $2[18,26]$ & 57 & N/A & 376 & N/A & 2 & 0 \\
\hline Vision-specific role difficulties & & & 2 & 57 & N/A & 376 & N/A & 1 & 1 \\
\hline VFQ-25 & 2 & N/A & $2[18,26]$ & 57 & N/A & 376 & N/A & $1[18]$ & $1[26]$ \\
\hline Vision-specific dependency & & & 2 & 57 & N/A & 376 & N/A & 2 & 0 \\
\hline VFQ-25 & 3 & N/A & $2[18,26]$ & 57 & N/A & 376 & N/A & 2 & 0 \\
\hline Vision-specific mental health & & & 2 & 57 & N/A & 376 & N/A & 2 & 0 \\
\hline VFQ-25 & 4 & N/A & $2[18,26]$ & 57 & N/A & 376 & N/A & 2 & 0 \\
\hline
\end{tabular}

Per complaint, each self-report measure used to describe this complaint is presented individually, along with the number of visual items it includes and the criterion that must be fulfilled for people to belong to the group with visual complaints. Weighted averages (prevalence of visual complaints) are presented for each complaint. References to the studies are placed in brackets []. ADLs, activities of daily living; CIRS-G, Cumulative Illness Rating Scale-Geriatric; CISS, Convergence Insufficiency Symptom Survey; COMPASS, Composite Autonomic Symptom Score; ICF, Internationa Classification of Functioning; N, number/population size; N/A, not available; NMSQ, Non-Motor Symptoms Questionnaire, NMSS = Non-Motor Symptom Scale, OSDI = Ocular Surface Disease Index, PD = Parkinson's disease, PRO-PD = Patient Reported outcomes in Parkinson's Disease; QoL, quality of life; SCOPA-AUT, SCales for Outcomes in PArkinson's disease-autonomic dysfunction; VAQ, Visual Activities Questionnaire; VFQ-25, Visual Function Questionnaire (25-item); VIPD-Q, Visual Impairment in Parkinson's Disease Questionnaire. a Difference in score or prevalence between the groups, ${ }^{b}$ Weighted prevalence of complaints on the ICF functions 'seeing functions' $(72.9 \%)$ and 'difficulty with watching' (78.2\%), ${ }^{\mathrm{c}}$ Items that may to some extent have arisen from motor symptoms (e.g., bumping into objects and freezing in narrow spaces) were included in the analysis. Prevalence was 57\% after removing these items, ${ }^{\mathrm{d}}$ Khedr et al. (2012) [90] $\&$ Khedr et al. (2013) [55], and Giorelli et al. (2014) [65] \& Giorelli et al. (2014) [66] used the same group of people with PD and reported the same results. For this reason, results of only one of these studies was reported, ${ }^{\mathrm{e}}$ Results only account for the non-tremor group $(N=12),{ }^{\mathrm{f}}$ Results only account for the tremor group $(N=13),{ }^{\mathrm{g}}$ Weighted prevalence of complaints on size $(17 \%)$ and shape perception (21\%), heighted prevalence of reading related complaints: 'letters disappear while reading' (20\% in patients and 5\% in controls) and 'read better with one eye closed' (17\% in patients and $6 \%$ in controls), ${ }^{\mathrm{i}}$ Weighted prevalence of the complaints freezing (60\% in patients and $3 \%$ in controls) and difficulty at doorways (56\% in patients and 3\% in controls) [119]. 
Table 3

Relationship of demographic and disease-related variables with visual complaints

\begin{tabular}{|c|c|c|c|c|}
\hline & Visual complaint & $\begin{array}{l}\text { Significant positive } \\
\text { relationship }\end{array}$ & $\begin{array}{l}\text { Significant } \\
\text { negative } \\
\text { relationship }\end{array}$ & $\begin{array}{l}\text { No significant } \\
\text { relationship }\end{array}$ \\
\hline \multicolumn{5}{|c|}{ Demographic or disease-related variable } \\
\hline \multirow[t]{4}{*}{ 1. Age } & Studies $(N)$ & 1 & 0 & 3 \\
\hline & Double vision & 0 & 0 & $2[66,111]$ \\
\hline & Increased sensitivity to light & 0 & 0 & $1[122]$ \\
\hline & Reduced visual health status & $1[26]$ & 0 & 0 \\
\hline \multirow{6}{*}{ 2. Gender $\left({ }^{+}\right.$men $>$women $)$} & Studies $(N)$ & 1 & 0 & 12 \\
\hline & Visual complaints in general & 0 & 0 & $1[117]$ \\
\hline & Double vision & 0 & 0 & $\begin{array}{l}10[34,50,66,67,72, \\
75,97,103,105,111]\end{array}$ \\
\hline & Increased sensitivity to light & 0 & 0 & $1[122]$ \\
\hline & Altered color vision & $1[17]$ & 0 & 0 \\
\hline & Difficulty estimating spatial relations & $1[17]^{\mathrm{a}}$ & 0 & 0 \\
\hline \multirow[t]{2}{*}{ 3. Education } & Studies $(N)$ & 0 & 0 & 1 \\
\hline & Double vision & 0 & 0 & $1[111]$ \\
\hline \multirow[t]{7}{*}{ 4. Disease duration } & Studies $(N)$ & 6 & 0 & 3 \\
\hline & Visual complaints in general & $2[32,117]$ & 0 & $1[17]$ \\
\hline & Double vision & $3[101,111,116]$ & 0 & $1[17]$ \\
\hline & Increased sensitivity to light & $1[137]$ & 0 & $1[122]$ \\
\hline & Altered color vision & 0 & 0 & $1[17]$ \\
\hline & Difficulty estimating spatial relations & 0 & 0 & $1[17]$ \\
\hline & Asthenopia & 0 & 0 & $1[152]$ \\
\hline \multirow[t]{9}{*}{ 5. Disease severity } & Studies $(N)$ & 12 & 0 & 6 \\
\hline & Visual complaints in general & 0 & 0 & $1[17]$ \\
\hline & Double vision & $5[52,59,110,111,116]$ & & $2[17,66]$ \\
\hline & Increased sensitivity to light & $4[120,132,138,151]^{\mathrm{b}}$ & & $4[122,131,133,151]^{\mathrm{c}}$ \\
\hline & Altered color vision & 0 & & $1[17]$ \\
\hline & Difficulty estimating spatial relations & 0 & & $1[17]$ \\
\hline & Asthenopia & $1[152]$ & 0 & 0 \\
\hline & Reduced visual health status & $2[18,26]$ & 0 & 0 \\
\hline & Bumping into objects & 0 & 0 & $1[17]$ \\
\hline \multirow[t]{2}{*}{ 6. Motor fluctuations } & Studies $(N)$ & 2 & 0 & 0 \\
\hline & Double vision & $2[104,111]$ & 0 & 0 \\
\hline \multirow{11}{*}{$\begin{array}{l}\text { 7. Dominant symptom } \\
\left({ }^{+} \text {PIGD }>\text { tremor dominant }\right)\end{array}$} & Studies $(N)$ & 2 & 0 & 7 \\
\hline & Double vision & $1[36]$ & 0 & $6[50,55,66,81,93,96]$ \\
\hline & Increased sensitivity to light & 0 & 0 & $1[142]$ \\
\hline & Altered color vision & 0 & 0 & $1[142]$ \\
\hline & Changes in depth perception & $1[142]$ & 0 & 0 \\
\hline & Reduced peripheral vision & 0 & 0 & $1[142]$ \\
\hline & Reduced light/dark adaptation & $1[142]$ & 0 & 0 \\
\hline & Reduced acuity/spatial vision & 0 & 0 & $1[142]$ \\
\hline & Reduced visual processing speed & 0 & 0 & $1[142]$ \\
\hline & Reduced visual search & 0 & 0 & $1[142]$ \\
\hline & Problems with visual ADLs & $1[142]$ & 0 & 0 \\
\hline \multirow[t]{2}{*}{ 8. Dyskinesia } & Studies $(N)$ & 1 & 0 & 0 \\
\hline & Double vision & $1[104]$ & 0 & 0 \\
\hline \multirow[t]{2}{*}{ 9. Left vs. right onset PD } & Studies $(N)$ & 0 & 0 & 1 \\
\hline & Double vision & 0 & 0 & $1[81]$ \\
\hline \multirow{2}{*}{$\begin{array}{l}\text { 10. Young vs. late onset PD } \\
\left({ }^{+} \text {late onset }>\text { young onset }\right)\end{array}$} & Studies $(N)$ & 2 & 0 & 2 \\
\hline & Double vision & $2[70,103]^{\mathrm{d}}$ & 0 & $2[77,79]$ \\
\hline \multirow{3}{*}{$\begin{array}{l}\text { 11. Idiopathic PD vs. } \\
\text { gene-related parkinsonism } \\
\text { (LRRK2 [33], G2385R [161] } \\
\text { and PARKIN [79]; } \\
\text { +idiopathic PD > gene-related } \\
\text { parkinsonism) }\end{array}$} & Studies $(N)$ & 1 & 0 & 3 \\
\hline & Double vision & $1[79]^{\mathrm{e}}$ & 0 & $2[33,79]^{\mathrm{fg}}$ \\
\hline & Increased sensitivity to light & 0 & 0 & $1[161]$ \\
\hline \multirow[t]{2}{*}{ 12. Camptocormia } & Studies $(N)$ & 1 & 0 & 1 \\
\hline & Double vision & $1[114]$ & 0 & $1[113]$ \\
\hline
\end{tabular}


Table 3

(Continued)

\begin{tabular}{|c|c|c|c|c|}
\hline & Visual complaint & $\begin{array}{l}\text { Significant positive } \\
\text { relationship }\end{array}$ & $\begin{array}{l}\text { Significant } \\
\text { negative } \\
\text { relationship }\end{array}$ & $\begin{array}{l}\text { No significant } \\
\text { relationship }\end{array}$ \\
\hline \multicolumn{5}{|l|}{ Medical or surgical treatment } \\
\hline \multirow[t]{4}{*}{ 13. LEDD } & Studies $(N)$ & 1 & 1 & 5 \\
\hline & Double vision & 0 & $1[111]$ & $1[54,59,82,94]$ \\
\hline & Reduced visual health status & $1[26]$ & 0 & 0 \\
\hline & Increased sensitivity to light & 0 & 0 & $1[132]$ \\
\hline \multirow{4}{*}{$\begin{array}{l}\text { 14. Off vs. on state }\left({ }^{+} \text {off }\right. \\
\text { state }>\text { on state })\end{array}$} & Studies $(N)$ & 3 & 0 & 0 \\
\hline & Visual complaints in general & $1[30]$ & 0 & 0 \\
\hline & Double vision & $1[111]$ & 0 & 0 \\
\hline & Problems focusing & $1[119]$ & 0 & 0 \\
\hline \multirow{3}{*}{$\begin{array}{l}\text { 15. Specific medical treatment } \\
\text { (Pergolide and Benzhexol } \\
\text { [119], and Rotigotine [ } 87 \text {, } \\
164] \text { ) }\end{array}$} & Studies $(N)$ & 1 & 0 & 2 \\
\hline & Double vision & 0 & 0 & $2[87,164]$ \\
\hline & Adverse visual effects & $1[119]^{\mathrm{h}}$ & 0 & 0 \\
\hline \multirow[t]{2}{*}{ 16. DBS (subthalamic nucleus) } & Studies $(N)$ & 0 & 2 & 0 \\
\hline & Double vision & 0 & $2[22,61]$ & 0 \\
\hline \multicolumn{5}{|l|}{ Other variables } \\
\hline \multirow{4}{*}{ 17. QoL and ADL } & Studies $(N)$ & 0 & 5 & 1 \\
\hline & Visual complaints in general & 0 & $1[117]$ & 0 \\
\hline & Double vision & 0 & $1[118]$ & 0 \\
\hline & Increased sensitivity to light & 0 & $3[122,137,151]$ & $1[125]$ \\
\hline \multirow{3}{*}{$\begin{array}{l}\text { 18. Consultation of medical } \\
\text { doctor for eye problem }\end{array}$} & Studies $(N)$ & 1 & 0 & 1 \\
\hline & Bumping into objects & $1[17]$ & 0 & 0 \\
\hline & Dry eyes & 0 & 0 & $1[162]$ \\
\hline
\end{tabular}

Supplementary Table 2 provides an overview of investigated relationships per complaint. $>$, first group reported more visual complaints than second group; ADL, activities of daily living; DBS, Deep Brain Stimulation; LEDD, Levodopa Equivalent Daily Dose; N, number of

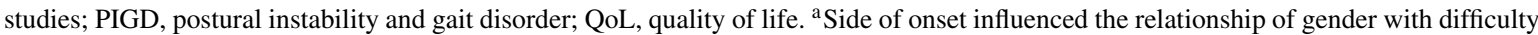
estimating spatial relations; the difference between males and females was bigger (and significant) for the left onset group than right onset group (not significant), b Disease severity measured by Hoehn \& Yahr classification and Clinical Impression of Severity Index for Parkinson's Disease (CISI-PD) [151], ${ }^{\mathrm{c}}$ Disease severity measured by Cumulative Illness Rating Scale - Geriatrics (CIRS-G) [151], ${ }^{\mathrm{d}}$ Only significant when adjusted for disease duration and dopaminergic treatment duration and dose [70], ${ }^{\mathrm{e}}$ Result accounts for a group of patients with late onset PD [79], ${ }^{\mathrm{f}}$ Result accounts for a group of patients with young onset PD [79], ${ }^{\mathrm{g}}$ Not significant when adjusted for age, gender, disease duration, disease severity, dopamine agonist, and levodopa [33], ${ }^{\mathrm{h}}$ More colorful dreams were reported after using Pergolide, and relaxation of the eye muscles and blurry vision after using Benzhexol [119].

\section{Function-related visual complaints}

\section{Visual complaints in general}

Seventeen studies [16-32] reported on visual complaints in general. Six of these studies compared people with PD with a control group. In all cases, the prevalence of visual complaints was higher among patients than among controls $[16,18,20,21,25$, 26]. One study examined the number of complaints experienced. Three out of four patients $(77.3 \%)$ experienced at least one complaint, and $43.2 \%$ experienced multiple visual complaints [25]. Only $31.6 \%$ of healthy controls reported a visual complaint [25].

\section{Double vision}

Sixty-one studies reported relevant results on double vision based on the Non-Motor Symptoms Questionnaire (NMSQ) [33-93] and 24 studies based on the Non-Motor Symptom Scale (NMSS) [9, 41, 94-115] (see Supplementary Figure 1). The prevalence ranged from $2.9 \%$ [72] to $23.5 \%$ [59] in studies with a sample size $\geq 150$. Studies with a smaller sample size $(N<150)$ showed a range from $0 \%[35,40$, 94] to $60.9 \%$ [45]. One study found that for most people with PD experiencing double vision $(9.6 \%$ of total sample) complaints started after motor symptoms had manifested. Only a small proportion $(1.8 \%$ of total sample) already experienced double vision before the onset of motor symptoms [71].

Most studies (15/18) on double vision showed a higher prevalence in people with PD compared to controls $[16,25,47,48,56,57,59,91,95,96,108$, 116-119]. One study reported prevalence numbers in more detail and showed that $16 \%$ of people with PD experienced double vision 'often', and 36\% 'sometimes' [118]. 


\section{Increased sensitivity to light}

Nineteen studies included the SCales for Outcomes in PArkinson's disease-autonomic dysfunction (SCOPA-AUT) [120] and reported relevant results for the item on oversensitivity to light in people with PD [120-138]. The prevalence ranged from $1.9 \%$ [130] to $61.0 \%$ [120] (see Supplementary Figure 2). Most studies (13/15) showed that the prevalence was higher in people with PD compared to controls $[25,117,119-121,123,128-130,132$, 138-140].

Other light-related complaints were more prevalent in people with PD as well. People with PD had significantly more difficulty adapting to changing light conditions than controls (patients: $27 \%$ vs. controls: $11 \%$ ) [117], and experienced reduced night vision more often (patients: $16 \%-47.6 \%$ vs. controls: $3 \%-18.5 \%[117,140])$.

\section{Altered color vision}

Prevalence of altered color vision did not significantly differ between people with PD and healthy controls in the majority of studies [18, 26, 118, 119, 141-143]. Three studies indicated a trend toward more complaints among people with PD [18, 26, $118]$. Only one study found that significantly more people with PD (11\%) reported the perception of colors to be more pale than controls did (3\%) [117]. Another study found that $10 \%$ of people with PD perceived less color than before, while $5 \%$ perceived more color [118].

\section{Painful, dry or watery eyes}

Complaints related to painful, dry or watery eyes were more frequently reported by people with PD than by controls (5/6 studies [18, 25, 117, 144, 145]). Also, severity seems to differ. People with PD had 'moderate dry eye disease' and controls 'mild dry eye disease' [145]. It was reported that $28 \%$ of people with PD had a burning sensation or gritty feeling in their eyes, and only $8 \%$ of controls [117]. While some studies could not confirm significance [25, 26], painful eyes were present in $27.3 \%$ of people with PD compared to $22.4 \%$ of controls [25]. Watery eyes were more frequently reported by people with PD $(26.8 \%-31 \%)$ than controls $(15 \%)$ [24, 117].

\section{Blurred vision}

The prevalence of blurry vision was higher in people with PD compared to healthy controls (4/4 studies [25, 117, 140, 146]). Patients experiencing blurry vision indicated that their vision was worse in the dark and they needed more light to see clearly [119].

\section{Changes in contrast sensitivity}

Changes in contrast sensitivity were more often reported by people with PD compared to controls $(2 / 2$ studies $[117,118])$. Roughly a quarter of people with PD $(27 \%)$ reported changes in the perception of contrast (23\% perceived less contrast, $4 \%$ perceived more contrast) [118]. People with PD (26\%) reported more difficulty reading plain text on a colored or gray background compared to controls (10\%) [117].

\section{Changes in size, shape, or depth perception}

People with PD reported more complaints regarding size, shape, or depth perception than controls [117, 119]. Seventeen percent of people with PD experienced difficulty with size, shape, and depth perception, compared to $2 \%$ (depth) - 5\% (size/shape) of controls [117].

\section{Peripheral vision}

People with PD experienced significantly more limitations in peripheral vision (i.e., "noticing objects off to the side') than controls $[18,26]$. People with PD $(43 \%)$ indicated to need to turn their head to the side to see peripheral objects more often than controls $\operatorname{did}(14 \%)$ [119].

\section{Difficulty estimating spatial relations}

Half of the patients $(50 \%)$ reported misjudging spaces (5\% 'often' and 45\% 'sometimes'), compared to $28 \%$ of controls [118]. Most people with PD $(27 \%)$ indicated that spaces appear smaller, while $17 \%$ noted that spaces seem larger.

\section{Visual field impairment}

People with PD experienced visual field impairments more often $(6.1 \%$ [24]-9\% [117]) than healthy controls (2\% [117]).

\section{Changes in motion perception}

Changes in motion perception were reported by a significantly higher number of people with PD (19\% $-25 \%$ ) than controls $(3 \%)[118,119]$. Eight percent of people with PD had difficulties 'often', and $17 \%$ 'sometimes'. Most people with PD perceived motion as faster (13\%), while few perceived motion to be slower (4\%). Others perceived motion to be less smooth (3\%) [118]. In addition, people with PD had more difficulty following quick movements with their eyes (patients: 25\% vs. controls: 4\%) [117]. 


\section{Activity-related visual complaints}

\section{Reading difficulties}

People with PD reported more difficulty reading than controls (4/4 studies [16, 25, 116, 117]). While one study could not confirm significance, a higher prevalence was found in people with PD $(26.7 \%)$ compared to controls (9.7\%) [16]. Compared to controls, people with PD reported significantly more often that letters disappeared while reading (patients: $20 \%$ vs. controls: $5 \%$ ) and that they had fewer reading difficulties when having one eye closed while reading (patients: 17\% vs. controls: 6\%) [117].

\section{Near and distant visual activities}

People with PD reported more problems with near (patients: $44.3 \%$ vs. controls: $25.5 \%$ ) and distant visual activities (patients: $38.6 \%$ vs. controls: $15.0 \%$ ) than controls [21]. All five studies confirmed this result $[18,21,26,147,148]$. Most individuals who had difficulty working nearby reported that their eyes felt tired or uncomfortable [147, 148]. In contrast, people with PD 'often' do not have trouble judging close $(95 \%)$ or distant objects $(87 \%)$, comparable to healthy controls, of which $96 \%$ and $99 \%$ did not have problems judging close or distant objects, respectively [118].

\section{Difficulty/freezing in narrow spaces}

People with PD had more difficulty in narrow spaces than controls (4/4 studies [25, 116, 118, 119]). Walking through narrow spaces was difficult for $70 \%$ of people with PD, compared to $1 \%$ of controls [118]. Eighteen percent of people with PD indicated to have difficulties 'often', and 52\% 'sometimes'. People with PD indicated to freeze (26\%), bump (29\%), feel uneasy $(10 \%)$, or have other problems (3\%) while walking through narrow spaces.

Freezing (patients: $60 \%$ vs. controls: $3 \%$ ) and difficulty at doorways (patients: $56 \%$ vs. controls: $3 \%$ ) was also significantly more common in people with PD compared to controls [119]. Half of the people with PD reported to bump into objects [17].

\section{Driving}

People with PD experienced more driving difficulties compared to controls $(3 / 3$ studies $[18,26$, 149]). This could be attributed to poor vision, especially at night. Along with that, people with PD reported difficulties with reading maps or using navigational devices [17]. Besides finding driving more difficult in low visibility conditions (patients: $21.6 \%$ vs. controls: $2.7 \%$ ), people with PD avoid these situations more frequently compared to controls (patients: $35.1 \%$ vs. controls: $5.4 \%$ ) [149].

\section{Difficulty reaching for objects}

People with PD reported significantly more difficulty reaching for objects (46\%) than controls (3\%). Nine percent of people with PD had difficulties 'often', and 37\% 'sometimes'. Most patients indicated to not reach far enough $(27 \%)$, while others reached too far $(7 \%)$ or reached off to one side of the object $(8 \%)$ [118].

\section{Vision-related QoL}

People with PD scored significantly worse than controls on vision-related QoL (Visual Function Questionnaire; VFQ [150]) [18, 26]. Social functioning and mental health are influenced, role difficulties exist, and people with PD are more dependent on others due to their visual problems. Except for role difficulties, vision-related QoL was influenced to a greater extent in people with PD than in controls.

\section{Relationship of demographic or disease-related variables with visual complaints}

Table 3 reports on the relationship of demographic and disease-related characteristics with self-reported visual complaints. Visual complaints did not relate to age (3/4 studies $[66,111,122])$ and gender $(12 / 13$ studies [34, 50, 66, 67, 72, 75, 97, 103, 105, 111, 117, $122])$. Most studies did find a relationship with disease duration $(6 / 9$ studies $[32,101,111,116,117$, 137]) and severity (12/18 studies [18, 26, 52, 59, $110,111,116,120,132,138,151,152])$, indicating that visual complaints were more prevalent in patients with a longer disease duration and a more severe disease. Dominant symptoms of the disease (7/9 studies $[50,55,66,81,93,96,142])$, as well as side of onset (1/1 study [81]) did not relate to visual complaints.

A higher Levodopa Equivalent Daily Dose (LEDD) was not related to visual complaints (5/7 studies [54, 59, 82, 94, 132]), while patients did report more complaints during off-state ( $3 / 3$ studies [30, 111, 119]). Some medicaments (i.e., Pergolide and Benzhexol) led to visual adverse effect (1/1 study [119]). Deep Brain Stimulation (DBS) was found to relieve double vision $(2 / 2$ studies [22, 61]).

The relationship of visual complaints with objectified visual disorders and non-motor symptoms was also investigated (see Supplementary Table 1). 
Double vision was related to abnormal ocular alignment and aberrant movements of the eyes [116]. Regarding painful or dry eyes, decreased tear production, abnormalities in Meibomian glands, and ocular surface problems could confirm the complaint [16, 145], but it was not related to decreased tear film break-up time or tear osmolarity [145]. Visual complaints were not related to cognitive functioning (3/4 studies $[102,111,151])$, but it did co-occur with psychotic symptoms (e.g., hallucinations; $3 / 3$ studies $[24,111,151])$.

Visual complaints were found to negatively relate to the performance of activities of daily living (ADL) and QoL (5/6 studies [117, 118, 122, 137, 151]).

Supplementary Table 2 presents an overview of investigated relationships with demographic and disease-related variables per visual complaint (an aid for understanding Table 3 and Supplementary Table 1).

\section{DISCUSSION}

This systematic review aimed to provide an overview of the literature on self-reported visual complaints in people with PD. Visual complaints occur in a wide variety in people with PD. Moreover, there is a fundamental difference between people with PD and healthy controls. More than $80 \%$ of comparisons showed that visual complaints were more common in people with PD than in controls.

According to the literature found, the prevalence of visual complaints varies greatly. Nevertheless, we can conclude that visual complaints were more often present in people with PD than in controls. Visual complaints in general appear to occur in at least a quarter of people with $\mathrm{PD}$, with prevalence ranging from $26.8 \%-98 \%$. Other complaints with a relatively high prevalence are blurred vision $(19.7 \%-46 \%)$, watery eyes $(26.8 \%-31 \%)$, light-related complaints (reduced night vision $(16 \%-47.6 \%)$ and light/dark adaptation $(27 \%)$ ), ocular fatigue $(43.6 \%)$, and all activity related complaints ( $>21.6 \%$; i.e., reading, driving, reaching, near or distant activities, and difficulty/freezing in narrow spaces). Complaints that appear less prevalent are altered color vision $(4.1 \%$ $12.7 \%)$, and visual field deficits $(6.1 \%-9 \%)$.

Vision-related QoL in people with PD appears to be affected in a variety of ways. This is not surprising given that vision is essential in performing a wide variety of activities. Moreover, people with PD need their vision to compensate for their motor loss [6]. Therefore, vision loss may lead to even more difficulties in daily life.

\section{Strengths and limitations}

We conducted a systematic literature search and selected all articles that met the inclusion criteria. By involving at least three researchers in the process of data extraction, we sought to ensure reliable and standardized data extraction.

We aimed at providing a comprehensive overview of the literature on self-reported visual complaints in PD. The inclusion of lower quality studies may have biased the results. The prevalence of a complaint was sometimes determined by only few studies and small sample sizes (e.g., asthenopia prevalence was determined in 39 patients [152]). Moreover, the studies used different methods, response options and criteria to assess the complaints in patient groups with different disease characteristics. Therefore, the results presented should be interpreted with caution and results cannot be directly compared. For example, the two lowest rates of double vision are found in de novo PD patients $[72,94]$, which is in line with the reported increase of visual complaints with disease duration and severity (see Table 3). Also, it was not always clear in the phrasing of questions that problems were attributed to vision [17, 118, 119]. People might have involved other symptoms when answering the questions (i.e., reading difficulties were attributed to both visual problems and difficulties with concentration [17]). For the sake of transparency, we listed the (number of) references, the number of participants in whom a complaint was investigated, and the criterion on which the prevalence was based.

Some visual complaints are reported relatively often, while there are also complaints that are hardly ever reported. An important explanation for this is that former complaints are addressed in frequently used self-reports or questionnaires, while the latter are not. This does not mean that visual complaints not included in those instruments are less common or should be taken less seriously. The majority of 139 included studies $(N=85,61.2 \%)$ reported double vision, an item in the NMSS and NMSQ. Similarly, oversensitivity to light constitutes an item in the SCOPA-AUT and was therefore reported in multiple studies $(N=19,13.7 \%)$. Only 35 articles $(25.2 \%)$ investigated visual complaints using other measures. For example, only few studies asked for experienced visual field loss or reduced contrast sensitivity, which might nevertheless be relevant in the light of known 
visual field and contrast sensitivity deficits in PD [4, 153]. To obtain a more complete picture of the prevalence, nature, and severity of visual complaints in people with $\mathrm{PD}$ and the characteristics of people who experience these complaints, future research should systematically examine a broad range of visual complaints in a large group of people with PD.

\section{Clinical implications}

Detection of visual complaints is necessary to prevent unnecessary limitations in QoL. With knowledge of the visual complaints a patient experiences, clinicians are able to select treatments based on the patient's priorities $[12,154]$. In addition, the use of self-report measures within rehabilitation may provide insight into the changes a patient is experiencing and can indicate when individual goals have been achieved [13].

Visual complaints may be an indication of a treatable underlying disorder, which requires assessment and treatment by an ophthalmologist. Complaints may also relate to underlying disorders that cannot be treated, in which case vision-related rehabilitation may be recommended. As an example, complaints of double vision may be caused by cataract, ptosis, or corneal surface abnormalities. These conditions may be treated with cataract extraction, surgery, or artificial tears, respectively. Another possible cause of double vision is convergence insufficiency, which may require prism glasses or advice on how to compensate while reading.

In some cases, objectified functional disorders may not fully reflect self-reported complaints. For example, even though color vision deficits are more common in people with PD compared to controls $[155,156]$, people with PD do not tend to report more complaints with regard to color vision. In this example, treatment seems irrelevant because patients do not suffer from the underlying disorder. Therefore, care should always be based on self-reported complaints as well.

Although the literature is scarce, visual complaints seem to be related to disease duration and severity. In addition, visual complaints might be influenced by certain types of treatment. Therefore, we suggest that people with PD should be questioned more frequently and more thoroughly about these complaints in clinical practice, especially in advanced disease stages or with medication changes.

The importance of active questioning is also evident from the fact that visual problems might not always be spontaneously reported to healthcare professionals $[48,49]$, given that people with PD and their family members are often unaware of the relationship between visual complaints and PD [50]. In addition, Martinez-Martin et al. (2007) argued that these complaints may not be recognized unless detected by a questionnaire [60]. Recently developed visual self-report questionnaires may be used to improve the visual care for people with PD [157, 158].

\section{Conclusion}

This overview illustrates that a substantial number of people with PD report complaints in a variety of visual domains. These complaints are related to disease duration and severity and may be influenced by medication use. Since visual complaints have a clear negative impact on QoL, attention to and management of these complaints is essential. Active questioning is advised for timely recognition and treatment of visual complaints.

\section{ACKNOWLEDGMENTS}

This work was supported by Stichting NOVUM, Amsterdam, The Netherlands, ZonMw grant 6370 05001 (Expertisefunctie Zintuiglijk Gehandicapten, Meerjarig deelsectorplan 2020-2022 Visueel).

Research support: E. Vooijs (BSc), Psychology student at the University of Groningen; K.C. Klaassen (MSc) and J.E.M. Meijerink (MSc), Patient-Researchers for the Dutch Parkinson's Disease Association.

\section{CONFLICT OF INTEREST}

The authors have no conflict of interest to report.

\section{SUPPLEMENTARY MATERIAL}

The supplementary material is available in the electronic version of this article: https://dx.doi.org/ 10.3233/JPD-202324.

\section{REFERENCES}

[1] Erro R, Santangelo G, Barone P, Vitale C (2015) Nonmotor symptoms in Parkinson's disease: Classification and management. J Park Restless Legs Syndr 5, 1-10.

[2] Pfeiffer RF (2016) Non-motor symptoms in Parkinson's disease. Parkinsonism Relat Disord 22(Suppl 1), S119S122. 
[3] Armstrong RA (2015) Oculo-visual dysfunction in Parkinson's disease. J Parkinsons Dis 5, 715-726.

[4] Weil RS, Schrag AE, Warren JD, Crutch SJ, Lees AJ, Morris HR (2016) Visual dysfunction in Parkinson's disease. Brain 139, 2827-2843.

[5] Diederich NJ, Raman R, Leurgans S, Goetz CG (2002) Progressive worsening of spatial and chromatic processing deficits in Parkinson disease. Arch Neurol 59, 1249-1252.

[6] Ekker MS, Janssen S, Seppi K, Poewe W, de Vries NM, Theelen T, Nonnekes J, Bloem BR (2017) Ocular and visual disorders in Parkinson's disease: Common but frequently overlooked. Parkinsonism Relat Disord 40, 1-10.

[7] Sauerbier A, Chaudhuri KR (2014) Non-motor symptoms: The core of multi-morbid Parkinson's disease. Br J Hosp Med (Lond) 75, 18-24.

[8] Armstrong RA (2008) Visual signs and symptoms of Parkinson's disease. Clin Exp Optom 91, 129-138.

[9] Martinez-Martin P, Rodriguez-Blazquez C, Kurtis MM, Chaudhuri KR (2011) The impact of non-motor symptoms on health-related quality of life of patients with Parkinson's disease. Mov Disord 26, 399-406.

[10] O'Sullivan SS, Williams DR, Gallagher DA, Massey LA, Silveira-Moriyama L, Lees AJ (2008) Nonmotor symptoms as presenting complaints in Parkinson's disease: A clinicopathological study. Mov Disord 23, 101-106.

[11] Shulman LM, Taback RL, Rabinstein AA, Weiner WJ (2002) Non-recognition of depression and other nonmotor symptoms in Parkinson's disease. Parkinsonism Relat Disord 8, 193-197.

[12] Reddy P, Martinez-Martin P, Brown RG, Chaudhuri KR, Lin JP, Selway R, Forgacs I, Ashkan K, Samuel M (2014) Perceptions of symptoms and expectations of advanced therapy for Parkinson's disease: Preliminary report of a Patient-Reported Outcome tool for Advanced Parkinson's disease (PRO-APD). Health Qual Life Outcomes 12, 11.

[13] Vingerhoets G, Lannoo E, van der Linden C, Caemaert J, Vandewalle V, van den Abbeele D, Wolters M (1999) Changes in quality of life following unilateral pallidal stimulation in Parkinson's disease. J Psychosom Res 46, 247-255.

[14] Moher D, Liberati A, Tetzlaff J, Altman DG, Altman D, Antes G, Atkins D, Barbour V, Barrowman N, Berlin JA, Clark J, Clarke M, Cook D, D'Amico R, Deeks JJ, Devereaux PJ, Dickersin K, Egger M, Ernst E, Gøtzsche PC, Grimshaw J, Guyatt G, Higgins J, Ioannidis JPA, Kleijnen J, Lang T, Magrini N, McNamee D, Moja L, Mulrow C, Napoli M, Oxman A, Pham B, Rennie D, Sampson M, Schulz KF, Shekelle PG, Tovey D, Tugwell $P$ (2009) Preferred reporting items for systematic reviews and meta-analyses: The PRISMA statement. PLoS Med 6, e1000097.

[15] Diederich NJ, Fénelon G, Stebbins G, Goetz CG (2009) Hallucinations in Parkinson disease. Nat Rev Neurol 5, 331-342.

[16] Biousse V, Skibell BC, Watts RL, Loupe DN, DrewsBotsch C, Newman NJ (2004) Ophthalmologic features of Parkinson's disease. Neurology 62, 177-180.

[17] Davidsdottir S, Cronin-Golomb A, Lee A (2005) Visual and spatial symptoms in Parkinson's disease. Vision Res 45, 1285-1296.

[18] Almer Z, Klein KS, Marsh L, Gerstenhaber M, Repka MX (2012) Ocular motor and sensory function in Parkinson's disease. Ophthalmology 119, 178-182.

[19] King LA, Priest KC, Nutt J, Chen Y, Chen Z, Melnick M HF (2014) Comorbidity and functional mobility in persons with Parkinson disease. Arch Phys Med Rehabil 95, 21522157.

[20] Schmidt C, Herting B, Prieur S, Junghanns S, Schweitzer K, Reichmann H, Berg D, Ziemssen T (2008) Autonomic dysfunction in patients with progressive supranuclear palsy. Mov Disord 23, 2083-2089.

[21] Hamedani AG, Willis AW (2020) Self-reported visual dysfunction in Parkinson disease: The Survey of Health, Ageing and Retirement in Europe. Eur J Neurol 27, 484-489.

[22] Kawaguchi M, Samura K, Miyagi Y, Okamoto T, Yamasaki R, Sakae N, Yoshida F, Iihara K (2020) The effects of chronic subthalamic stimulation on nonmotor symptoms in advanced Parkinson's disease, revealed by an online questionnaire program. Acta Neurochir (Wien) 162, 247-255.

[23] Aggarwal R, Goyal V, Pandey RM, Kumar N, Singh S, Shukla G, Behari M (2020) Development and validation of quality of life in Parkinson's disease instrument. Ann Indian Acad Neurol 23, 59-71.

[24] Marques A, Beze S, Pereira B, Chassain C, Monneyron N, Delaby L, Lambert C, Fontaine M, Derost P, Debilly B, Rieu I, Lewis SJG, Chiambaretta F, Durif F (2020) Visual hallucinations and illusions in Parkinson's disease: The role of ocular pathology. J Neurol 267, 2829-2841.

[25] Urwyler P, Nef T, Killen A, Collerton D, Thomas A, Burn D, McKeith I, Mosimann UP (2014) Visual complaints and visual hallucinations in Parkinson's disease. Parkinsonism Relat Disord 20, 318-322.

[26] Brandt AU, Zimmermann HG, Oberwahrenbrock T, Isensee J, Müller T, Paul F (2018) Self-perception and determinants of color vision in Parkinson's disease. $\mathrm{J} \mathrm{Neu}$ ral Transm (Vienna) 125, 145-152.

[27] Raggi A, Leonardi M, Ajovalasit D, Carella F, Soliveri P, Albanese A, Romito L (2011) Disability and profiles of functioning of patients with Parkinson's disease described with ICF classification. Int J Rehabil Res 34, 141-150.

[28] Abudi S, Ma RN (1997) Parkinson's disease symptoms patients' perceptions. J Adv Nurs 25, 54-59.

[29] Schenkman M, Cutson TM, Zhu CW, Whetten-Goldstein K (2002) A longitudinal evaluation of patients' perceptions of Parkinson's disease. Gerontologist 42, 790-798.

[30] Witjas T, Kaphan E, Azulay JP, Blin O, Ceccaldi M (2002) Nonmotor fluctuations in Parkinson's disease. Neurology 59, 408-413.

[31] Whitson HE, Sanders LL, Pieper CF, Morey MC, Oddone EZ, Gold DT, Cohen HJ (2009) Correlation between symptoms and function in older adults with comorbidity. J Am Geriatr Soc 57, 676-682.

[32] Mischley LK, Lau RC, Weiss NS (2017) Use of a selfrating scale of the nature and severity of symptoms in Parkinson's Disease (PRO-PD): Correlation with quality of life and existing scales of disease severity. NPJ Parkinsons Dis 3, 20.

[33] Li DW, Gu Z, Wang C, Ma J, Tang BS, Chen S Di, Chan $P$ (2015) Non-motor symptoms in Chinese Parkinson's disease patients with and without LRRK2 G2385R and R1628P variants. J Neural Transm (Vienna) 122, 661-667.

[34] Durcan R, Wiblin L, Lawson RA, Khoo TK, Yarnall AJ, Duncan GW, Brooks DJ, Pavese N, Burn DJ (2019) Prevalence and duration of non-motor symptoms in prodromal Parkinson's disease. Eur J Neurol 26, 979-985.

[35] Ragab OA, Elheneedy YA, Bahnasy WS (2019) Nonmotor symptoms in newly diagnosed Parkinson's disease patients. Egypt J Neurol Psychiatry Neurosurg 55, 1-7. 
[36] Ba F, Obaid M, Wieler M, Camicioli R, Martin WRW (2015) Parkinson disease: The relationship between nonmotor symptoms and motor phenotype. Can J Neurol Sci 43, 261-267.

[37] Moccia M, Mollenhauer B, Erro R, Picillo M, Palladino $\mathrm{R}$, Barone P (2015) Non-motor correlates of smoking habits in de novo Parkinson's disease. J Parkinsons Dis $\mathbf{5}$, 913-924.

[38] Erro R, Picillo M, Vitale C, Amboni M, Moccia M, Santangelo G, Pellecchia MT, Barone P (2016) The non-motor side of the honeymoon period of Parkinson's disease and its relationship with quality of life: A 4-year longitudinal study. Eur J Neurol 23, 1673-1679.

[39] Tanveer K, Attique I, Sadiq W, Ahmad A (2018) Nonmotor symptoms in patients with Parkinson's disease: A cross-sectional survey. Cureus 10, e3412.

[40] Aldaz T, Nigro P, Sánchez-Gómez A, Painous C, Planellas L, Santacruz P, Cámara A, Compta Y, Valldeoriola F, Martí MJ, Muñoz E (2019) Non-motor symptoms in Huntington's disease: A comparative study with Parkinson's disease. J Neurol 266, 1340-1350.

[41] Salari M, Chitsaz A, Etemadifar M, Najafi MR, Mirmosayyeb O, Bemanalizadeh M, Panahi F, Mirzajani $H$ (2017) Evaluation of non-motor symptoms and their impact on quality of life in patients with Parkinson's disease, Isfahan, Iran. Iran J Neurol 16, 118-124.

[42] Liu WM, Wu RM, Lin CH, Tai CH, Yu RL, Lin RJ (2015) The impact of nonmotor symptoms on quality of life in patients with Parkinson's disease in Taiwan. Neuropsychiatr Dis Treat 11, 2865-2873.

[43] Duncan GW, Khoo TK, Yarnall AJ, O'Brien JT, Coleman SY, Brooks DJ, Barker RA, Burn DJ (2014) Health-related quality of life in early Parkinson's disease: The impact of nonmotor symptoms. Mov Disord 29, 195-202.

[44] Gan J, Zhou M, Chen W, Liu Z (2014) Non-motor symptoms in Chinese Parkinson's disease patients. J Clin Neurosci 21, 751-754.

[45] Lolekha P, Kulkantrakorn K (2014) Non-motor symptoms in Thai Parkinson's disease patients: Prevalence, manifestation and health related quality of life. Neurol Asia 19, 163-170.

[46] Barone P, Antonini A, Colosimo C, Marconi R, Morgante L, Avarello TP, Bottacchi E, Cannas A, Ceravolo G, Ceravolo R, Cicarelli G, Gaglio RM, Giglia RM, Iemolo F, Manfredi M, Meco G, Nicoletti A, Pederzoli M, Petrone A, Pisani A, Pontieri FE, Quatrale R, Ramat S, Scala R, Volpe G, Zappulla S, Bentivoglio AR, Stocchi F, Trianni G, Del Dotto P; PRIAMO study group (2009) The PRIAMO study: A multicenter assessment of nonmotor symptoms and their impact on quality of life in Parkinson's disease. Mov Disord 24, 1641-1649.

[47] Bostantjopoulou S, Katsarou Z, Karakasis C, Peitsidou E, Milioni D, Rossopoulos N (2013) Evaluation of non-motor symptoms in Parkinson's disease: An underestimated necessity. Hippokratia 17, 214-219.

[48] Chaudhuri KR, Martinez-Martin P, Schapira AH, Stocchi F, Sethi K, Odin P, Brown RG, Koller W, Barone P, MacPhee G, Kelly L, Rabey M, MacMahon D, Thomas S, Ondo W, Rye D, Forbes A, Tluk S, Dhawan V, Bowron A, Williams AJ, Olanow CW (2006) International multicenter pilot study of the first comprehensive self-completed nonmotor symptoms questionnaire for Parkinson's disease: The NMSQuest study. Mov Disord 21, 916-923.

[49] Chaudhuri KR, Prieto-Jurcynska C, Naidu Y, Mitra T, Frades-Payo B, Tluk S, Ruessmann A, Odin P, Macphee
G, Stocchi F, Ondo W, Sethi K, Schapira AH, Martinez Castrillo JC, Martinez-Martin P (2010) The nondeclaration of nonmotor symptoms of Parkinson's disease to health care professionals: An international study using the nonmotor symptoms questionnaire. Mov Disord 25, 704-709.

[50] Cheon SM, Ha MS, Park MJ, Kim JW (2008) Nonmotor symptoms of Parkinson's disease: Prevalence and awareness of patients and families. Parkinsonism Relat Disord 14, 286-290.

[51] Cosentino C, Nuñez Y, Torres L (2013) Frecuencia de síntomas no motores en Pacientes peruanos con enfermedad de Parkinson. Arq Neuropsiquiatr 71, 216-219.

[52] Crosiers D, Pickut B, Theuns J, Deyn PP, Van Broeckhoven C, Martinez-Martin P, Chaudhuri KR, Cras P (2012) Non-motor symptoms in a Flanders-Belgian population of 215 Parkinson's disease patients as assessed by the nonmotor symptoms questionnaire. Am J Neurodegener Dis $\mathbf{1}$, 160-167.

[53] Erro R, Santangelo G, Picillo M, Vitale C, Amboni M, Longo K, Costagliola A, Pellecchia MT, Allocca R, De Rosa A, De Michele G, Santoro L, Barone P (2012) Link between non-motor symptoms and cognitive dysfunctions in de novo, drug-naive PD patients. J Neurol 259, 18081813.

[54] Erro R, Picillo M, Vitale C, Amboni M, Moccia M, Longo K, Cozzolino A, Giordano F, De Rosa A, De Michele G, Pellecchia MT, Barone P (2013) Non-motor symptoms in early Parkinson's disease: A 2-year follow-up study on previously untreated patients. J Neurol Neurosurg Psychiatry 84, 14-17.

[55] Khedr EM, El Fetoh NA, Khalifa H, Ahmed MA, El Beh KM (2013) Prevalence of non motor features in a cohort of Parkinson's disease patients. Clin Neurol Neurosurg 115, 673-677.

[56] Mao CJ, Chen JP, Zhang XY, Chen Y, Li SJ, Li J, Xiong KP, Hu WD, Liu CF (2015) Parkinson's disease patients with pain suffer from more severe non-motor symptoms. Neurol Sci 36, 263-268.

[57] Khoo TK, Yarnall AJ, Duncan GW, Coleman S, O'Brien JT, Brooks DJ, Barker RA, Burn DJ (2013) The spectrum of nonmotor symptoms in early Parkinson disease. Neurology 80, 176-181.

[58] Li HJ, Zhang MF, Chen MX, Hu AL, Li J Bin, Zhang B, Liu W (2015) Validation of the nonmotor symptoms questionnaire for Parkinson's disease: Results from a Chinese pilot study. Int J Neurosci 125, 929-935.

[59] Zhang N, Liu W, Ye M, Cohen AD, Zhang Y (2015) The heterogeneity of non-motor symptoms of Parkinson's disease. Neurol Sci 36, 577-584.

[60] Martinez-Martin P, Schapira AH, Stocchi F, Sethi K, Odin P, MacPhee G, Brown RG, Naidu Y, Clayton L, Abe K, Tsuboi Y, MacMahon D, Barone P, Rabey M, Bonuccelli U, Forbes A, Breen K, Tluk S, Olanow CW, Thomas S, Rye D, Hand A, Williams AJ, Ondo W, Chaudhuri KR (2007) Prevalence of nonmotor symptoms in Parkinson's disease in an international setting; study using nonmotor symptoms questionnaire in 545 patients. Mov Disord 22, 1623-1629.

[61] Nazzaro JM, Pahwa R, Lyons KE (2011) The impact of bilateral subthalamic stimulation on non-motor symptoms of Parkinson's disease. Parkinsonism Relat Disord 17, 606-609.

[62] Raggi A, Leonardi M, Carella F, Soliveri P, Albanese A, Romito LM (2011) Impact of nonmotor symptoms 
on disability in patients with Parkinson's disease. Int $J$ Rehabil Res 34, 316-320.

[63] Yu B, Xiao ZY, Li JZ, Yuan J, Yi-Ming L (2010) Study of an integrated non-motor symptoms questionnaire for Parkinson's disease. Chin Med J (Engl) 123, 1436-1440.

[64] Rios Romenets S, Wolfson C, Galatas C, Pelletier A, Altman R, Wadup L, Postuma RB (2012) Validation of the non-motor symptoms questionnaire (NMS-Quest). Parkinsonism Relat Disord 18, 54-58.

[65] Giorelli M, Bagnoli J, Consiglio L, Difazio P, Zizza D, Zimatore GB (2014) Change in non-motor symptoms in Parkinson's disease and essential tremor patients: A oneyear follow-up study. Tremor Other Hyperkinet Mov (N Y) 4, 216.

[66] Giorelli M, Bagnoli J, Consiglio L, Lopane M, Bosco Zimatore G, Zizza D, Difazio P (2014) Do non-motor symptoms in Parkinson's disease differ from essential tremor before initial diagnosis? A clinical and scintigraphic study. Parkinsonism Relat Disord 20, 17-21.

[67] Sánchez-Martínez CM, Choreño-Parra JA, PlacenciaÁlvarez N, Nuñez-Orozco L, Guadarrama-Ortiz P (2019) Frequency and dynamics of non-motor symptoms presentation in Hispanic patients with Parkinson disease. Front Neurol 10, 1197.

[68] Vongvaivanich K, Nidhinandana S, Udommongkol C, Chairungsaris P, Chinvarun Y, Wongmek W, Suphakasem S, Suwantamee J, Sithinamsuwan P (2014) Non-motor symptoms in Thai patients with Parkinson's disease studied at Phramongkutklao Hospital. J Med Assoc Thai 97, S159-S167.

[69] Sengul Y, Sengul HS, Sural MK, Bakim B, Forta H (2015) A comparison between rate of nonmotor symptom development in essential tremor and Parkinson's disease. Acta Neurol Belg 115, 289-294.

[70] Zhou MZ, Gan J, Wei YR, Ren XY, Chen W, Liu ZG (2013) The association between non-motor symptoms in Parkinson's disease and age at onset. Clin Neurol Neurosurg 115, 2103-2107.

[71] Zhang TM, Yu SY, Guo P, Du Y, Hu Y, Piao YS, Zuo LJ, Lian TH, Wang RD, Yu QJ, Jin Z, Zhang W (2016) Nonmotor symptoms in patients with Parkinson disease: A cross-sectional observational study. Medicine (Baltimore) 95, e5400.

[72] Picillo M, Amboni M, Erro R, Longo K, Vitale C, Moccia M, Pierro A, Santangelo G, De Rosa A, De Michele G, Santoro L, Orefice G, Barone P, Pellecchia MT (2013) Gender differences in non-motor symptoms in early, drug naive Parkinson's disease. J Neurol 260, 2849-2855.

[73] Wang G, Wan Y, Cheng Q, Xiao Q, Wang Y, Zhang J, Ma JF, Wang XJ, Zhou HY, Chen SD (2010) Malnutrition and associated factors in Chinese patients with Parkinson's disease: Results from a pilot investigation. Parkinsonism Relat Disord 16, 119-123.

[74] Cubo E, López Peña MJ, Diez-Feijo Varela E, Pérez Gil O, Garcia Gutierrez P, Araus González E, Prieto Tedejo R, Mariscal Pérez N, Armesto D (2014) Lack of association of morphologic and functional retinal changes with motor and non-motor symptoms severity in Parkinson's disease. J Neural Transm (Vienna) 121, 139-145.

[75] Mukhtar S, Imran R, Zaheer M, Tariq H (2018) Frequency of non-motor symptoms in Parkinson's disease presenting to tertiary care centre in Pakistan: An observational, crosssectional study. BMJ Open 8, e019172.

[76] Rukmini Mridula K, Borgohain R, Jabeen SA, Padmaja G, Bandaru VS, Ankathi P, Kanikannan MA, Ali Khan MS
(2015) Comparison of frequencies of non motor symptoms in Indian Parkinson's disease patients on medical management versus deep brain stimulation: A case-control study. Iran J Neurol 14, 86-93.

[77] Špica V, Pekmezović T, Svetel M, Kostić VS (2013) Prevalence of non-motor symptoms in young-onset versus late-onset Parkinson's disease. J Neurol 260, 131-137.

[78] Gulunay A, Cakmakli GY, Yon MI, Ulusoy EK, Karakoc M (2020) Frequency of non-motor symptoms and their impact on the quality of life in patients with Parkinson's disease: A prospective descriptive case series. Psychogeriatrics 20, 206-211.

[79] Kägi G, Klein C, Wood NW, Schneider SA, Pramstaller PP, Tadic V, Quinn NP, van de Warrenburg BP, Bhatia KP (2010) Nonmotor symptoms in Parkin gene-related Parkinsonism. Mov Disord 25, 1279-1284.

[80] Erro R, Picillo M, Amboni M, Moccia M, Vitale C, Longo K, Pellecchia MT, Santangelo G, Martinez-Martin P, Ray Chaudhuri K, Barone P (2015) Nonmotor predictors for levodopa requirement in de novo patients with Parkinson's disease. Mov Disord 30, 373-378.

[81] Rodríguez-Violante M, Cervantes-Arriaga A, VillarVelarde A, Corona T (2011) Relationship between the type and side of motor symptoms with the prevalence of nonmotor symptoms in Parkinson's disease. Neurologia 26, 319-324.

[82] Picillo M, Erro R, Amboni M, Longo K, Vitale C, Moccia M, Pierro A, Scannapieco S, Santangelo G, Spina E, Orefice G, Barone P, Pellecchia MT (2014) Gender differences in non-motor symptoms in early Parkinson's disease: A 2-years follow-up study on previously untreated patients. Parkinsonism Relat Disord 20, 850-854.

[83] Guan X, Wang Y, Li Q, Wei M, Chen L, Cheng O (2018) Analysis of the clinical features of early Parkinson's disease with comparatively integrated intestinal function. Neurol Sci 39, 1847-1856.

[84] Mekawichai P, Kunadisorn S, Tungkasereerak C, Saetang S (2016) Non-motor symptoms in Thai Parkinson's disease patients and the correlation with motor symptoms. Neurol Asia 21, 41-46.

[85] Neikrug AB, Avanzino JA, Liu L, Maglione JE, Natarajan L, Corey-Bloom J, Palmer BW, Loredo JS, Ancoli-Israel S (2014) Parkinson's disease and REM sleep behavior disorder result in increased non-motor symptoms. Sleep Med 15, 959-966.

[86] Prell T, Schaller D, Perner C, Witte OW, Grosskreutz J (2020) Sicca symptoms in Parkinson's disease: Association with other nonmotor symptoms and health-related quality of life. Parkinsons Dis 2020, 2958635.

[87] Valldeoriola F, Salvador A, Gómez-Arguelles JM, Marey J, Moya M, Ayuga Á, Ramírez F (2018) The effects of transdermal rotigotine on nonmotor symptoms of Parkinson's disease: A multicentre, observational, retrospective, postmarketing study. Int J Neurosci 128, 369-375.

[88] Yu QJ, Yu SY, Zuo LJ, Lian TH, Hu Y, Wang RD, Piao YS, Guo P, Liu L, Jin Z, Li LX, Chan P, Chen S Di, Wang XM, Zhang W (2018) Parkinson disease with constipation: Clinical features and relevant factors. Sci Rep 8, 567.

[89] Samart K (2018) Non-motor symptoms in Thai Parkinson's disease patients: Prevalence and associated factors. Neurol Asia 23, 327-331.

[90] Khedr EM, Abo El Fetoh N, Khalifa H, Ahmed MA, El Beh KMA (2012) Prevalence of depression, anxiety, dementia and other non motor features of a large cohort 
of Egyptian Parkinson's disease patients. Life Sci J 9, 509-518.

[91] Zhong LL, Song YQ, Cao H, Ju KJ, Yu L (2021) The non-motor symptoms of Parkinson's disease of different motor types in early stage. Eur Rev Med Pharmacol Sci 21, 5745-5750.

[92] Govorova T, Popova T, Tappakhov A, Andreev M (2019) Assessment of non-motor symptoms in essential tremor. Int J Biomed 9, 308-312.

[93] Ren J, Hua P, Pan C, Li Y, Zhang L, Zhang W, Xu P, Zhang M, Liu W (2020) Non-motor symptoms of the postural instability and gait difficulty subtype in de novo Parkinson's disease patients: A cross-sectional study in a single center. Neuropsychiatr Dis Treat 16, 2605-2612.

[94] Kim HJ, Park SY, Cho YJ, Hong KS, Cho JY, Seo SY, Lee DH, Jeon BS (2009) Nonmotor symptoms in de novo Parkinson disease before and after dopaminergic treatment. J Neurol Sci 287, 200-204.

[95] Kim J, Youn J, Shin H, Cho J (2013) Nonmotor symptoms in drug-induced parkinsonism and drug-naïve Parkinson disease. Can J Neurol Sci 40, 36-41.

[96] Wu Y, Guo XY, Wei QQ, Ou RW, Song W, Cao B, Zhao B, Shang HF (2016) Non-motor symptoms and quality of life in tremor dominant vs postural instability gait disorder Parkinson's disease patients. Acta Neurol Scand 133, 330-337.

[97] Martinez-Martin P, Falup Pecurariu C, Odin P, van Hilten JJ, Antonini A, Rojo-Abuin JM, Borges V, Trenkwalder C, Aarsland D, Brooks DJ, Ray Chaudhuri K (2012) Genderrelated differences in the burden of non-motor symptoms in Parkinson's disease. J Neurol 259, 1639-1647.

[98] Song W, Guo X, Chen K, Chen X, Cao B, Wei Q, Huang R, Zhao B, Wu Y, Shang HF (2014) The impact of non-motor symptoms on the health-related quality of life of Parkinson's disease patients from Southwest China. Parkinsonism Relat Disord 20, 149-152.

[99] Shalash AS, Hamid E, Elrassas HH, Bedair AS, Abushouk AI, Khamis M, Hashim M, Ahmed NS, Ashour S, Elbalkimy M (2018) Non-motor symptoms as predictors of quality of life in Egyptian patients with Parkinson's disease: A cross-sectional study using a culturally adapted 39-item Parkinson's disease questionnaire. Front Neurol 9, 357.

[100] Ou R, Yang J, Cao B, Wei Q, Chen K, Chen X, Zhao B, Wu Y, Song W, Shang H (2016) Progression of nonmotor symptoms in Parkinson's disease among different age populations: A two-year follow-up study. J Neurol Sci 360, 72-77.

[101] Guo X, Song W, Chen K, Chen X, Zheng Z, Cao B, Huang R, Zhao B, Wu Y, Shang HF (2013) Disease durationrelated differences in non-motor symptoms: A study of 616 Chinese Parkinson's disease patients. J Neurol Sci 330, 32-37.

[102] Zhang SS, Ou RW, Chen XP, Yang J, Zhao B, Yuan XQ, Wei QQ, Cao B, Shang HF (2016) Correlative factors of cognitive dysfunction in PD patients: A cross-sectional study from Southwest China. Neurol Res 38, 434-440.

[103] Guo X, Song W, Chen K, Chen XP, Zheng Z, Cao B, Huang R, Zhao B, Wu Y, Shang HF (2013) Gender and onset age-related features of non-motor symptoms of patients with Parkinson's disease - a study from Southwest China. Parkinsonism Relat Disord 19, 961-965.

[104] Santos-García D, de Deus Fonticoba T, Suárez Castro E, Aneiros Díaz A, McAfee D, Catalán MJ, Alonso-Frech F, Villanueva C, Jesús S, Mir P, Aguilar M, Pastor P, García
Caldentey J, Esltelrich Peyret E, Planellas LL, Martí MJ, Caballol N, Hernández Vara J, Martí Andrés G, Cabo I, Ávila Rivera MA, López Manzanares L, Redondo N, Martinez-Martin P; COPPADIS Study Group, McAfee D (2020) Non-motor symptom burden is strongly correlated to motor complications in patients with Parkinson's disease. Eur J Neurol 27, 1210-1223.

[105] Ravan A, Ahmad FM, Chabria S, Gadhari M, Sankhla CS (2015) Non-motor symptoms in an Indian cohort of Parkinson's disease patients and correlation of progression of non-motor symptoms with motor worsening. Neurol India 63, 166-174.

[106] Weerkamp NJ, Tissingh G, Poels PJ, Zuidema SU, Munneke M, Koopmans RT, Bloem BR (2013) Nonmotor symptoms in nursing home residents with Parkinson's disease: Prevalence and effect on quality of life. J Am Geriatr Soc 61, 1714-1721.

[107] Cho BH, Choi SM, Kim JT, Kim BC (2018) Association of coffee consumption and non-motor symptoms in drugnaïve, early-stage Parkinson's disease. Parkinsonism Relat Disord 50, 42-47.

[108] Santos García D, Jesús S, Aguilar M, Planellas LL, García Caldentey J, Caballol N, Legarda I, Hernández Vara J, Cabo I, López Manzanares L, González Aramburu I, Ávila Rivera MA, Catalán MJ, López Díaz L, Puente V, García Moreno JM, Borrué C, Solano Vila B; Álvarez Sauco M, Vela L, Escalante S, Cubo E, Carrillo Padilla F, Martínez Castrillo JC, Sánchez Alonso P, Alonso Losada MG, López Ariztegui N, Gastón I, Kulisevsky J, Menéndez González M, Seijo M, Rúiz Martínez J, Valero C, Kurtis M, de Fábregues-Boixar O, González Ardura J, Prieto Jurczynska C, Martinez-Martin P, Mir P; COPPADIS Study Group (2019) COPPADIS-2015 (COhort of Patients with PArkinson's DIsease in Spain, 2015): An ongoing global Parkinson's disease project about disease progression with more than 1000 subjects included. Results from the baseline evaluation. Eur J Neurol 26, 1399-1407.

[109] Rosqvist K, Odin P, Hagell P, Iwarsson S, Nilsson MH, Storch A (2019) Dopaminergic effect on non-motor symptoms in late stage Parkinson's disease. J Parkinsons Dis 9 , 447-448.

[110] Toyama M, Okuma Y, Yamamoto M, Kashihara K, Yoshida K, Saiki H, Maeda T, Tsuboi Y, Takahashi Y, Nakayama T (2020) Non-motor symptoms depending on motor severity in Japanese patients with Parkinson's disease: A multicenter cross-sectional study. J Neurol Sci 412, 116641.

[111] Schindlbeck KA, Schönfeld S, Naumann W, Friedrich DJ, Maier A, Rewitzer C, Klostermann F, Marzinzik F (2017) Characterization of diplopia in non-demented patients with Parkinson's disease. Parkinsonism Relat Disord 45, 1-6.

[112] Karri M, Ramasamy B, Kalidoss R (2020) Prevalence of non-motor symptoms in Parkinson's disease and its impact on quality of life in tertiary care center in India. Ann Indian Acad Neurol 23, 270-274.

[113] Ou R, Guo X, Song W, Cao B, Wei Q, Shao N, Zhao B, Shang H (2014) Characteristics of non-motor symptoms in patients with Parkinson's disease exhibiting camptocormia. Gait Posture 40, 447-450.

[114] Ou R, Liu H, Hou Y, Song W, Cao B, Wei Q, Yuan X, Chen Y, Zhao B, Shang H (2018) Predictors of camptocormia in patients with Parkinson's disease: A prospective study from southwest China. Parkinsonism Relat Disord 52, 69-75. 
[115] Santos-García D, de la Fuente-Fernández R (2013) Impact of non-motor symptoms on health-related and perceived quality of life in Parkinson's disease. J Neurol Sci 332, 136-140.

[116] Archibald NK, Clarke MP, Mosimann UP, Burn DJ (2011) Visual symptoms in Parkinson's disease and Parkinson's disease dementia. Mov Disord 26, 2387-2395.

[117] Borm CDJM, Visser F, Werkmann M, de Graaf D, Putz D, Seppi K, Poewe W, Vlaar AMM, Hoyng C, Bloem BR, Theelen T, de Vries NM (2020) Seeing ophthalmologic problems in Parkinson disease: Results of a visual impairment questionnaire. Neurology 94, e1539-e1547.

[118] Lee A, Harris J (1999) Problems with perception of space in Parkinson's disease: A questionnaire study. Neuroophthalmology 22, 1-15.

[119] McDowell SA, Harris JP (1997) Visual problems in Parkinson's disease: A questionnaire survey. Behav Neurol 10, 77-81.

[120] Visser M, Marinus J, Stiggelbout AM, van Hilten JJ (2004) Assessment of autonomic dysfunction in Parkinson's disease: The SCOPA-AUT. Mov Disord 19, 1306-1312.

[121] Bostantjopoulou S, Katsarou Z, Danglis I, Karakasis H, Milioni D (2016) Self-reported autonomic symptoms in Parkinson's disease: Properties of the SCOPA-AUT scale. Hippokratia 20, 115-120.

[122] Tomic S, Rajkovaca I, Pekic V, Salha T, Misevic S (2017) Impact of autonomic dysfunctions on the quality of life in Parkinson's disease patients. Acta Neurol Belg 117, 207-211.

[123] Mollenhauer B, Trautmann E, Sixel-Döring F, Wicke T, Ebentheuer J, Schaumburg M, Lang E, Focke NK, Kumar KR, Lohmann K, Klein C, Schlossmacher MG, Kohnen R, Friede T, Trenkwalder C (2013) Nonmotor and diagnostic findings in subjects with de novo Parkinson disease of the DeNoPa cohort. Neurology 81, 1226-1234.

[124] Kim R, Jun JS (2019) Association of autonomic symptoms with presynaptic striatal dopamine depletion in drug-naive Parkinson's disease: An analysis of the PPMI data. Auton Neurosci 216, 59-62.

[125] Merola A, Romagnolo A, Rosso M, Suri R, Berndt Z, Maule S, Lopiano L, Espay AJ (2018) Autonomic dysfunction in Parkinson's disease: A prospective cohort study. Mov Disord 33, 391-397.

[126] Sklerov M, Shih CH, Browner N, Palma JA, Styner M, Dayan E (2020) Longitudinal change in autonomic symptoms predicts activities of daily living and depression in Parkinson's disease. Clin Auton Res 30, 223-230.

[127] van Deursen DN, van den Heuvel OA, Booij J, Berendse HW, Vriend C (2020) Autonomic failure in Parkinson's disease is associated with striatal dopamine deficiencies. J Neurol 267, 1922-1930.

[128] Sprenger FS, Seppi K, Djamshidian A, Reiter E, Nocker M, Mair K, Göbel G, Poewe W (2015) Nonmotor symptoms in subjects without evidence of dopaminergic deficits. Mov Disord 30, 976-981.

[129] Garg D, Srivastava AK, Jaryal AK, Rajan R, Singh A, Pandit AK, Vibha D, Shukla G, Garg A, Pandey RM, Prasad $\mathrm{K}$ (2020) Is there a difference in autonomic dysfunction between multiple system atrophy subtypes? Mov Disord Clin Pract 7, 405-412.

[130] Stanković I, Petrović I, Pekmezović T, Marković V, Stojković T, Dragašević-Mišković N, Svetel M, Kostić V (2019) Longitudinal assessment of autonomic dysfunction in early Parkinson's disease. Parkinsonism Relat Disord 66, 74-79.
[131] Mantarova SG, Velcheva IV, Georgieva SO, Stambolieva KI (2013) Validation of the Bulgarian version of Scales for Outcomes in Parkinson's Disease - Autonomic (SCOPAAUT-BG). Folia Med (Plovdiv) 55, 56-62.

[132] Verbaan D, Marinus J, Visser M, Van Rooden SM, Stiggelbout AM, Van Hilten JJ (2007) Patient-reported autonomic symptoms in Parkinson disease. Neurology 69, 333-341.

[133] Malek N, Lawton MA, Grosset KA, Bajaj N, Barker RA, Burn DJ, Foltynie T, Hardy J, Morris HR, Williams NM, Ben-Shlomo Y, Wood NW, Grosset DG (2017) Autonomic dysfunction in early Parkinson's disease: Results from the United Kingdom tracking Parkinson's study. Mov Disord Clin Pract 4, 509-516.

[134] Valentino F, Bartolotta TV, Cosentino G, Mastrilli S, Arnao V, Aridon P, Scurria S, Pavone A, Pavone C, D'Amelio M (2018) Urological dysfunctions in patients with Parkinson's disease: Clues from clinical and noninvasive urological assessment. BMC Neurol 18, 148.

[135] Arnao V, Cinturino A, Valentino F, Perini V, Mastrilli S, Bellavia G, Savettieri G, Realmuto S, D'Amelio M (2015) In patient's with Parkinson disease, autonomic symptoms are frequent and associated with other non-motor symptoms. Clin Auton Res 25, 301-307.

[136] Kurtis MM, Rodriguez-Blazquez C, Martinez-Martin P; ELEP Group (2013) Relationship between sleep disorders and other non-motor symptoms in Parkinson's disease. Parkinsonism Relat Disord 19, 1152-1155.

[137] Tibar H, El Bayad K, Bouhouche A, Ait Ben Haddou EH, Benomar A, Yahyaoui M, Benazzouz A, Regragui W (2018) Non-motor symptoms of Parkinson's disease and their impact on quality of life in a cohort of Moroccan patients. Front Neurol 9, 170.

[138] Damian A, Adler CH, Hentz JG, Shill HA, Caviness JN, Sabbagh MN, Evidente VG, Beach TG, Driver-Dunckley E (2012) Autonomic function, as self-reported on the SCOPA-autonomic questionnaire, is normal in essential tremor but not in Parkinson's disease. Parkinsonism Relat Disord 18, 1089-1093.

[139] Frauscher B, Nomura T, Duerr S, Ehrmann L, Gschliesser $\mathrm{V}$, Wenning GK, Wolf E, Inoue Y, Högl B, Poewe W (2012) Investigation of autonomic function in idiopathic REM sleep behavior disorder. J Neurol 259, 10561061.

[140] Reimann M, Schmidt C, Herting B, Prieur S, Junghanns S, Schweitzer K, Globas C, Schoels L, Reichmann H, Berg D, Ziemssen T (2010) Comprehensive autonomic assessment does not differentiate between Parkinson's disease, multiple system atrophy and progressive supranuclear palsy. $J$ Neural Transm (Vienna) 117, 69-76.

[141] Gaenslen A, Swid I, Liepelt-Scarfone I, Godau J, Berg D (2011) The patients' perception of prodromal symptoms before the initial diagnosis of Parkinson's disease. Mov Disord 26, 653-658.

[142] Seichepine DR, Neargarder S, Miller IN, Riedel TM, Gilmore GC, Cronin-Golomb A (2011) Relation of Parkinson's disease subtypes to visual activities of daily living. J Int Neuropsychol Soc 17, 841-852.

[143] Zimmermann M, Gaenslen A, Prahl K, Srulijes K, Hauser AK, Schulte C, Csoti I, Berg D, Brockmann K (2019) Patient's perception: Shorter and more severe prodromal phase in GBA-associated PD. Eur J Neurol 26, 694-698.

[144] Tamer C, Melek IM, Duman T, Öksüz H (2005) Tear film tests in Parkinson's disease patients. Ophthalmology 112, 1795. 
[145] Nowacka B, Lubinski W, Honczarenko K, Potemkowski A, Safranow K (2014) Ophthalmological features of Parkinson disease. Med Sci Monit 20, 2243-2249.

[146] Bulpitt CJ, Shaw K, Clifton P, Stern G, Davies JB, Reid JL (1985) The symptoms of patients treated for Parkinson's disease. Clin Neuropharmacol 8, 175-183.

[147] Law C, Chriqui E, Kergoat MJ, Leclerc BS, Panisset M, Irving EL, Postuma RB, Chouinard S, Kergoat H (2017) Prevalence of convergence insufficiency-type symptomatology in Parkinson's disease. Can J Neurol Sci 44, 562-566.

[148] Irving EL, Chriqui E, Law C, Kergoat MJ, Leclerc BS, Panisset M, Postuma R, Kergoat H (2017) Prevalence of convergence insufficiency in Parkinson's disease. Mov Disord Clin Pract 4, 424-429.

[149] Stolwyk RJ, Scally KA, Charlton JL, Bradshaw JL, Iansek R, Georgiou-Karistianis N (2015) Self-regulation of driving behavior in people with Parkinson disease. Cogn Behav Neurol 28, 80-91.

[150] Mangione CM, Berry S, Spritzer K, Janz NK, Klein R, Owsley C, Lee PP (1998) Identifying the content area for the 51-item national eye institute visual function questionnaire: Results from focus groups with visually impaired persons. Arch Ophthalmol 116, 227-233.

[151] Rodriguez-Blazquez C, Forjaz MJ, Frades-Payo B, de Pedro-Cuesta J, Martinez-Martin P; Longitudinal Parkinson's Disease Patient Study, Estudio Longitudinal de Pacients con Enfermedad da Parkinson Group (2010) Independent validation of the scales for outcomes in Parkinson's disease-autonomic (SCOPA-AUT). Eur $J$ Neurol 17, 194-201.

[152] Repka MX, Claro MC, Loupe DN, Reich SG (1996) Ocular motility in Parkinson's disease. J Pediatr Ophthalmol Strabismus 33, 144-147.

[153] Yenice O, Onal S, Midi I, Ozcan E, Temel A, I-Gunal D (2008) Visual field analysis in patients with Parkinson's disease. Parkinsonism Relat Disord 14, 193-198.

[154] Hobart JC, Freeman JA, Lamping DL (1996) Physician and patient-oriented outcomes in progressive neurological disease: Which to measure? Curr Opin Neurol 9, 441-444.

[155] Piro A, Tagarelli A, Nicoletti G, Fletcher R, Quattrone A (2014) Color vision impairment in Parkinson's disease. $J$ Parkinsons Dis 4, 317-319.
[156] Birch J, Kolle RU, Kunkel M, Paulus W, Upadhyay P (1998) Acquired colour deficiency in patients with Parkinson's disease. Vision Res 38, 3421-3426.

[157] Huizinga F, Heutink J, de Haan GA, van der Lijn I, van der Feen FE, Vrijling ACL, Melis-Dankers BJM, de Vries SM, Tucha O, Koerts J (2020) The development of the Screening of Visual Complaints questionnaire for patients with neurodegenerative disorders: Evaluation of psychometric features in a community sample. PLoS One 15, e0232232.

[158] Borm CDJM, Werkmann M, Visser F, Peball M, Putz D, Seppi K, Poewe W, Notting IC, Vlaar A, Theelen T, Hoyng C, Bloem BR, de Vries NM (2019) Towards seeing the visual impairments in Parkinson's disease: Protocol for a multicentre observational, cross-sectional study. BMC Neurol 19, 141.

[159] Kleiner-Fisman G, Martine R, Lang AE, Stern MB (2011) Development of a non-motor fluctuation assessment instrument for Parkinson disease. Parkinsons Dis 2011, 292719.

[160] Mathur S, Mursaleen L, Stamford J, Dewitte S, Robledo I, Isaacs T (2017) Challenges of improving patient-centred care in Parkinson's disease. J Parkinsons Dis 7, 163-174.

[161] Yang J, Wang H, Yuan Y, Fan S, Li L, Jiang C, Mao C, Shi C, Xu Y (2021) Peripheral synucleinopathy in Parkinson disease with LRRK2 G2385R variants. Ann Clin Transl Neurol 8, 592-602.

[162] Leclair-Visonneau L, Clairembault T, Volteau C, Chapelet G, Le Dily S, Vavasseur F, Coron E, Préterre C, Neunlist M, Péréon Y, Derkinderen P (2019) Colonic neuropathology is not associated with autonomic dysfunction in Parkinson's disease. Parkinsonism Relat Disord 61, 224-247.

[163] Charlton JL, Oxley J, Fildes B, Oxley P, Newstead S, Koppel S, O'Hare M (2006) Characteristics of older drivers who adopt self-regulatory driving behaviours. Transp Res Part F Traffic Psychol Behav 9, 363-373.

[164] Antonini A, Bauer L, Dohin E, Oertel WH, Rascol O, Reichmann H, Schmid M, Singh P, Tolosa E, Chaudhuri KR (2015) Effects of rotigotine transdermal patch in patients with Parkinson's disease presenting with nonmotor symptoms - results of a double-blind, randomized, placebo-controlled trial. Eur J Neurol 22, 1400-1407. 